\title{
Consenso chileno SOCHINEP-SER para el diagnóstico y tratamiento del asma en el escolar ${ }^{\wedge}$
}

\author{
ANA MARÍA HERRERA G. ${ }^{1,2}$ (COORDINADORA), SELIM ABARA E. ${ }^{3,4,5}$, CECILIA ÁLVAREZ G. ${ }^{6,7}$, \\ CLAUDIA ASTUDILLO M. ${ }^{8,9,10}$, RAÚL CORRALES V..$^{6,7}$, EVELYN CHALA C. ${ }^{2,11}$, \\ CECILIA DASZENIES S. ${ }^{12}$, ANDRÉS KOPPMANN A. ${ }^{5,13,14}$, VIVIANA LEZANA S. ${ }^{15}$, \\ ROSSANA MAMANI J. ${ }^{1,2}$, MARÍA ANGÉLICA PÉREZ H. ${ }^{1}$ y CRISTINA PIERRY V. ${ }^{6,7}$
}

\section{Chilean SOCHINEP-SER consensus for asthma diagnosis and treatment} in school age children

Bronchial asthma is the most prevalent chronic condition among children, however, in Chile, it is underdiagnosed. This may be due to medical professionals failing to recognize the disease. It is essential to be aware of the symptoms and signs that are suggestive of the disease in order to begin an appropriate treatment to achieve disease control. Asthma must be suspected in school age children who present repeated episodes of bronchial obstruction. The diagnosis should be confirmed with lung function tests that demonstrate variable airflow obstruction with a positive bronchodilator response. Treatment is based on two fundamental pillars: education and pharmacological treatment. Educational activities must include: information about the disease and its treatment, regular monitoring of treatment adherence, teaching and reviewing the correct inhalation technique at every checkup, developing a personalized written action plan and scheduling regular follow-up appointments. The gold standard for treatment is maintenance inhaled corticosteroids, in the lowest possible dose that enables disease control. The goal of the treatment is to eliminate daily symptoms and asthma crisis. Therapy should be increased if control is not achieved, but before starting it, adherence to maintenance treatment, inhalation technique, presence of associated comorbidities and environmental exposure should be evaluated. In the mild patient, who is not receiving maintenance therapy, rescue treatment should be done with bronchodilators, always associated with inhaled corticosteroids. This consensus is a guide to improve the diagnosis, treatment and control of asthma in schoolchildren

Key words: Asthma; children; diagnosis; treatment.

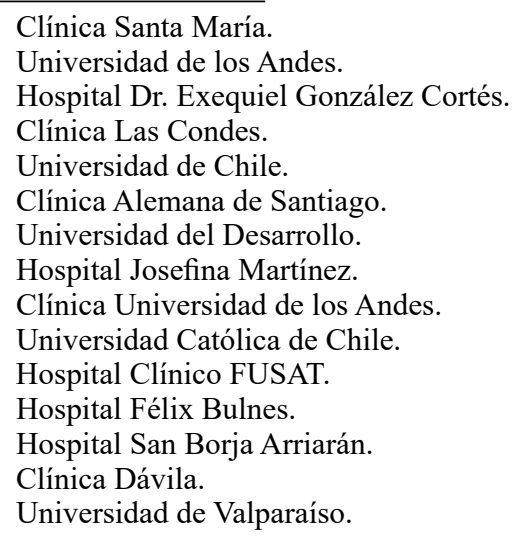

${ }^{\wedge}$ Esta es una publicación conjunta de la Sociedad Chilena de Neumología Pediátrica y de la Sociedad Chilena de Enfermedades Respiratorias en Revista Neumología Pediátrica y Revista Chilena de Enfermedades Respiratorias.

Los autores declaran no tener conflictos de interés en relación a esta publicación. 


\section{Resumen}

El asma bronquial es la enfermedad crónica más frecuente en la infancia. Sin embargo, en Chile existe un importante subdiagnóstico. Es fundamental estar atentos a los síntomas y signos que nos hacen sospechar el diagnóstico para iniciar un tratamiento oportuno, que asegure un buen control de la enfermedad. Debemos sospechar asma en todo escolar que presente cuadros repetidos de obstrucción bronquial. El diagnóstico debe confirmarse con pruebas de función pulmonar que demuestren obstrucción variable al flujo aéreo y respuesta broncodilatadora positiva. El tratamiento se basa en dos pilares fundamentales: la educación y el tratamiento farmacológico. Las actividades educativas deben incluir contenidos acerca de la enfermedad y su tratamiento, se debe monitorizar constantemente la adherencia al tratamiento de mantención, enseñar la técnica inhalatoria correcta y revisar en cada control, entregar un plan de acción escrito personalizado frente al inicio de una crisis y realizar controles médicos periódicos. Con respecto al tratamiento farmacológico, el estándar de oro es el uso de corticoides inhalados permanentes, en la mínima dosis posible que logre el control de la enfermedad. El objetivo del tratamiento es la supresión de los síntomas diarios y de las crisis. El tratamiento se irá incrementando en la medida que no haya una respuesta adecuada, pero antes de aquello se debe evaluar la adherencia al tratamiento de mantención, la técnica inhalatoria, presencia de comorbilidades asociadas y exposición ambiental. En el paciente leve, que esté sin tratamiento permanente, el rescate debe realizarse con broncodilatadores asociados siempre a un corticoide inhalado. Este consenso es una guía de apoyo para mejorar el diagnóstico oportuno, tratamiento y control del asma en el escolar.

Palabras clave: Asma; niños; diagnóstico; tratamiento.

\section{Introducción}

Según la Organización Mundial de la Salud (OMS), el asma bronquial es un problema sanitario de alta prevalencia a nivel mundial. Se calcula que 235 millones de personas están afectadas por esta enfermedad y que anualmente mueren $383.000^{1}$. El asma bronquial es la enfermedad no transmisible más frecuente en la infancia y la adolescencia, causando gran ausentismo escolar y deterioro de la calidad de vida del paciente y de su familia, junto a elevados costos en salud. Un adecuado control de la enfermedad permite revertir esta situación en forma importante. De acuerdo a The International Study of Asthma and Allergies in Childhood (ISAAC) la prevalencia de asma en Chile es de 17,9\% en niños de 6-7 años y de $15,3 \%$ en niños de $13-14^{2}$. En el último tiempo se ha observado un aumento muy significativo de las tasas de hospitalización por asma en niños chilenos desde 3,7 por 10.000 habitantes el año 2001 a 7,8 por 10.000 habitantes el 2014, verificándose además un importante subdiagnóstico de la enfermedad ${ }^{3,4}$.

\section{Definición}

Enfermedad inflamatoria crónica de las vías aéreas, caracterizada por episodios recurrentes de obstrucción bronquial. Es una patología heterogénea que incluye a diversos fenotipos que comparten manifestaciones clínicas comunes, pero de fisiopatología diferente. Se asocia a una hiperreactividad de la vía aérea independientemente del tipo de inflamación presente.

\section{Patogenia}

Los distintos fenotipos inflamatorios descritos en la literatura son: eosinofílico, neutrofílico y paucicelular, siendo el primero el predominante en la edad pediátrica ${ }^{5}$. El asma alérgico eosinofílico es mediado por la activación de linfocitos $\mathrm{T}$ Helper 2 y se caracteriza por la producción de interleucina 4, 5 y 13 (IL4, IL 5, IL13), reclutamiento de eosinófilos y niveles elevados de inmunoglobulina $\mathrm{E}$ ( $\mathrm{IgE}$ ) en sangre. Este fenotipo alérgico aparece desde los primeros años de vida y se asocia a una buena respuesta al tratamiento con corticoides inhalados ${ }^{6}$.

\section{Diagnóstico}

El diagnóstico es esencialmente clínico, pero siempre debe confirmarse con exámenes de laboratorio.

\section{Diagnóstico clínico}

Es fundamental una anamnesis detallada puesto que otras condiciones y enfermedades crónicas pueden tener manifestaciones clínicas similares. Los síntomas habituales son tos, sibilancias, dificultad para respirar, ahogo, cansancio y/o 
sensación de pecho apretado. Los principales desencadenantes de crisis de asma son las infecciones respiratorias virales, alérgenos intra y extradomiciliarios, contaminantes ambientales y ejercicio. Los síntomas se intensifican característicamente en la noche y al despertar y su alivio con tratamiento broncodilatador es muy sugerente de asma bronquial. Es importante evaluar la frecuencia y duración de los síntomas, la presencia de síntomas en períodos intercríticos y su interferencia con las actividades de la vida diaria y la calidad de vida. Deben consignarse los antecedentes de hospitalizaciones por asma en los últimos 12 meses, necesidad de consultas en servicio de urgencia, uso de corticoides sistémicos y tratamiento de mantención recibido, lo que permite categorizar la severidad de la enfermedad. Son importantes tam- bién los antecedentes personales de dermatitis atópica, alergia alimentaria y rinitis alérgica y en los padres la presencia de asma bronquial y rinitis. El examen físico en pacientes asintomáticos es habitualmente normal. Durante los períodos de pérdida de control y mientras ocurre una crisis de asma se pueden encontrar signos de hiperinsuflación pulmonar con aumento del diámetro anteroposterior del tórax e hipersonoridad a la percusión; a la auscultación pulmonar puede haber disminución del murmullo pulmonar, espiración prolongada y sibilancias espiratorias difusas. Sin embargo, es importante considerar que las sibilancias pueden estar presentes en otras enfermedades de la vía aérea (Tabla 1). Al examen físico pueden encontrarse además signos de dermatitis atópica y de rinitis alérgica ${ }^{5-7}$.

Tabla 1. Diagnóstico diferencial de asma en el niño

\begin{tabular}{|l|l}
\hline Clave diagnóstica & Posible diagnóstico \\
\hline Historia perinatal y familiar &
\end{tabular}

Síntomas desde recién nacido o patología pulmonar perinatal

Historia familiar de enfermedad pulmonar inusual

Enfermedades de la vía aérea

\section{Síntomas y signos}

Tos productiva persistente

Vómitos excesivos

Disfagia

Llanto o voz anormal

Signos focalizados al examen del tórax

Estridor inspiratorio y espiratorio

Bajo incremento pondoestatural

Hipocratismo digital

Alteraciones radiológicas focales o persistentes

Soplo cardiaco, cardiomegalia, insuficiencia cardiaca

Pólipos nasales

Diarrea crónica

Neumonía recurrente
Fibrosis quística, displasia broncopulmonar, malformaciones pulmonares congénitas, disquinesia ciliar primaria, inmunodeficiencias

Fibrosis quística, anomalías del desarrollo esquelético, enfermedades neuromusculares

Anomalías congénitas y adquiridas de la vía aérea, traqueomalacia

Fibrosis quística, reflujo gastroesofágico, trastorno de deglución, cuerpo extraño, disquinesia ciliar primaria, inmunodeficiencias, bronquiectasias, tuberculosis (TBC).

Reflujo gastroesofágico, aspiración

Problema de deglución, aspiración

Patología laríngea

Anomalías del desarrollo, bronquiolitis obliterante, bronquiectasias, TBC, cuerpo extraño, tumores

Patología laríngea o problemas de vía aérea central extratorácica, disfunción de cuerdas vocales, anillo vascular

Fibrosis quística, inmunodeficiencia, reflujo gastroesofágico

Fibrosis quística, bronquiectasias, bronquiolitis obliterante, disquinesia ciliar primaria, cardiopatías congénitas

Bronquiolitis obliterante, aspiración recurrente, cuerpo extraño en vía aérea, bronquiectasias, atelectasias, TBC, malformaciones congénitas pulmonares

Cardiopatía congénita

Fibrosis quística

Fibrosis quística, inmunodeficiencias

Inmunodeficiencia, fibrosis quística, aspiración pulmonar, disquinesia ciliar primaria, cuerpo extraño bronquial 


\section{Exámenes de laboratorio}

\section{a) Exámenes de función pulmonar}

\section{Espirometría basal y post broncodilatador}

Todo paciente con sospecha de asma bronquial debe tener una espirometría basal y con broncodilatador de acción corta (salbutamol). Esto permite evaluar la limitación al flujo aéreo espiratorio y la reversibilidad total o parcial a dicha limitación con el uso del broncodilatador. Este examen debe ser realizado idealmente en condiciones estables, de manera de obtener una medición basal de referencia y debe cumplir con criterios de aceptabilidad y reproducibilidad. Es indispensable contar con un equipo profesional calificado y estándares de calidad certificados que garanticen exactitud y reproducibilidad de los exámenes de función pulmonar. El informe y la interpretación de los resultados es labor del médico.

Los parámetros a evaluar son: capacidad vital forzada $(\mathrm{CVF})$, volumen espiratorio forzado de primer segundo $\left(\mathrm{VEF}_{1}\right)$, la relación $\mathrm{VEF}_{1} / \mathrm{CVF}$ y flujo espiratorio forzado entre el $25 \%$ y el $75 \%$ de la capacidad vital forzada (FEF 25\%-75\%). Para cada parámetro de función pulmonar, se considera normal un valor sobre el percentil 5 del valor predicho o sobre el limite inferior de la normalidad (LIN), utilizando como valores normales de referencia las ecuaciones multiétnicas Global Lung Function Initiative (GLI) $2012^{8}$. Se considera alteración ventilatoria obstructiva a una relación $\mathrm{VEF}_{1} / \mathrm{CVF}$ bajo el LIN, con un $\mathrm{VEF}_{1}$ que puede estar normal o bajo el $\mathrm{LIN}^{9,11}$. En algunos casos de limitación obstructiva moderada a severa se puede tener una CVF disminuida.

La respuesta broncodilatadora se evalúa aplicando $400 \mu \mathrm{g}$ (4 puffs) de salbutamol en inhalador de dosis medida, mediante un espaciador adecuado y repitiendo la espirometría $15 \mathrm{~min}$ después. Un aumento del $\mathrm{VEF}_{1} \geq 12 \%$ respecto del valor pre-broncodilatador constituye una respuesta broncodilatadora positiva y apoya el diagnóstico de asma. Respecto al FEF 25\%-75\%, el cambio con broncodilatador tiene menor poder diagnóstico y se considera cambio significativo $\geq 30 \%$ sin modificación de la $\mathrm{CVF}^{10}$. Los pacientes con un $\mathrm{VEF}_{1}$ disminuido especialmente bajo $60 \%$ del teórico y aquellos con respuesta broncodilatadora positiva, se consideran con mayor riesgo de crisis futura. En pacientes con sospecha clínica de asma y una limitación al flujo aéreo no reversible o fija se debe realizar una prueba terapéutica con prednisona $1 \mathrm{mg} / \mathrm{kg} /$ día, máximo $40 \mathrm{mg}$ /día por 5 a 10 días para evaluar reversibilidad. Un cambio $\geq 15 \%$ respecto al valor previo, en $\mathrm{VEF}_{1}$ y $\mathrm{CVF}$ puede considerarse significati$\mathrm{vo}^{12}$. Si no hay reversibilidad debe reevaluarse el diagnóstico de asma o considerar remodelación de la vía aérea con limitación ventilatoria obstructiva persistente.

Se ha demostrado que un $75 \%$ de los niños con asma persistente moderada a grave tienen un patrón de crecimiento pulmonar anormal con declinación precoz de la función pulmonar y con mayor riesgo de desarrollar enfermedad pulmonar obstructiva crónica (EPOC) en la vida adulta ${ }^{13}$. La espirometría no solo es útil para confirmar el diagnóstico de asma sino también para evaluar el control de la enfermedad, la respuesta al tratamiento, el riesgo de crisis de asma y la monitorización periódica de la función pulmonar (Tabla 2). Es importante destacar que una espirometría basal normal no descarta el diagnóstico de asma.

\section{Flujo espiratorio máximo (FEM)}

La medición del flujo espiratorio máximo (FEM) es muy dependiente del dispositivo utilizado y del esfuerzo del paciente. Se realiza con un flujómetro portátil fácilmente disponible en servicios de urgencia, consultas y en domicilio. Puede apoyar el diagnóstico de asma y es una herramienta de ayuda en el seguimiento de pacientes con asma grave, mal controlada o en pacientes con mala percepción de sus síntomas. Permite documentar la reversibilidad de la limitación del flujo aéreo espiratorio midiendo el FEM posterior al broncodilatador. Se considera una respuesta positiva un aumento de $20 \%$ del FEM pre broncodilatador. También es posible monitorizar la variabilidad diaria del FEM diurno y nocturno durante períodos largos de tiempo o después de realizar ejercicio. Se considera limitación variable al flujo espiratorio un cambio $>13 \%$ del FEM matinal y vespertino medido 2 veces al día por 2 semanas. Se calcula de la siguiente forma:

(FEM máximo día - FEM mínimo del día) $\bullet 100$ (FEM máximo + FEM mínimo) / 2

Tabla 2. Indicaciones de la espirometría en niños con sospecha clínica de asma

Medición inicial al momento del diagnóstico y previo al inicio del tratamiento

Si la espirometría inicial está alterada, controlar dentro de los primeros 3 a 6 meses de tratamiento 5,9

Control espirométrico cada 1 a 2 años ${ }^{5,9}$

Asma no controlada

Antes de suspender tratamiento de mantención 
Pruebas de hiperreactividad de la vía aérea

Si no se ha podido documentar la limitación variable del flujo aéreo o existe duda diagnó se recomienda realizar pruebas de provocación bronquial para determinar la presencia de hiperreactividad bronquial:

\section{Prueba de provocación bronquial con metacolina}

Este examen tiene una alta sensibilidad, pero baja especificidad ya que se ha descrito una hiperrespuesta de vía aérea a metacolina en pacientes con rinitis alérgica, fibrosis quística y displasia broncopulmonar. Una prueba de metacolina no reactiva es útil para excluir el diagnóstico de asma cuando hay síntomas presentes, ya que su valor predictivo negativo es mayor que su valor predictivo positivo. Esta prueba evalúa la concentración $\left(\mathrm{PC}_{20}\right)$ o dosis $\left(\mathrm{PD}_{20}\right)$ de metacolina necesaria para provocar una caída del $20 \%$ del $\mathrm{VEF}_{1}$ basal. Actualmente no existen consensos nacionales ni internacionales para valores normales de referencia de $\mathrm{PC}_{20}$ o $\mathrm{PD}_{20}$ en niños y los puntos de corte son arbitrarios. La Sociedad Chilena de Neumología Pediátrica (SOCHINEP) sugiere utilizar los puntos de corte recomendados por la European Respiratory Society (ERS). Esta considera una prueba de metacolina no reactiva a una $\mathrm{PC}_{20}>16 \mathrm{mg} / \mathrm{ml}\left(\mathrm{PD}_{20}>400 \mu \mathrm{g}\right)$ y una prueba de metacolina reactiva, compatible con hiperreactividad bronquial, a una $\mathrm{PC}_{20}<4 \mathrm{mg} /$ $\mathrm{ml}\left(\mathrm{PD}_{20}<100 \mu \mathrm{g}\right)$. Valores de $\mathrm{PC}_{20}$ entre 4-16 $\mathrm{mg} / \mathrm{ml}\left(\mathrm{PD}_{20} 100-400 \mu \mathrm{g}\right)$ se consideran valores limítrofes de reactividad bronquial ${ }^{14-17}$.

\section{Prueba de provocación bronquial con ejercicio}

Este examen tiene una alta especificidad, pero baja sensibilidad, siendo útil para confirmar el diagnóstico de asma especialmente en niños con síntomas inducidos por ejercicio. Se considera una prueba positiva una caída ${ }^{3} 10 \%$ del $\mathrm{VEF}_{1}$ o bien una caída $>15 \%$ del FEM respecto de su valor pre-ejercicio dentro de los $30 \mathrm{~min}$ luego de finalizado el ejercicio. Se recomienda medir la respuesta al ejercicio con $\mathrm{VEF}_{1}$ y no con FEM debido a la mayor reproducibilidad del primero ${ }^{17-20}$.

\section{b) Exámenes para evaluar inflamación de vía aérea y atopia}

La presencia de atopía aumenta la probabilidad de tener un asma alérgico, con inflamación eosinofílica. Su presencia no es específica de asma ni está presente en todos los fenotipos de asma. Los siguientes exámenes están disponibles para evaluar atopía aérea:
Pruebas cutáneas (prick test) o medición de IgE especificas en suero

El examen de pruebas cutáneas, prick test, es simple, rápido, de bajo costo y alta sensibilidad. Se mide la sensibilidad a alérgenos inhalantes intradomiciliarios y extradomiciliarios. Los alérgenos alimentarios no son un factor precipitante común de los síntomas del asma. La medición de $\operatorname{IgE}$ específicas no es superior a las pruebas cutáneas y son de alto costo. Se recomiendan en casos muy seleccionados como pacientes no cooperadores, presencia de dermatitis atópica extensa, tratamiento permanente con antihistamínicos o historia sugerente de anafilaxis. Se debe ponderar la importancia de las pruebas positivas (pápula igual o mayor a $3 \mathrm{~mm}$ de diámetro sobre el valor del control negativo) siempre en el contexto de la historia clínica del paciente. Estas pruebas son además de utilidad para el manejo educativo de factores gatillantes de crisis asmática.

\section{Medición directa de la inflamación de la vía aérea}

La determinación del tipo de inflamación de la vía aérea en forma directa se realiza por medio de estudio citológico del esputo inducido o muestras de lavado broncoalveolar y/o biopsia bronquial. No es un examen de rutina y se realiza solo en pacientes con asma bronquial grave y en centros especializados.

\section{Medición indirecta de la inflamación de la vía aérea \\ Fracción exhalada de óxido nitrico (FeNO)}

El FeNO se encuentra elevado en el asma con inflamación eosinofílica de la vía aérea, sin embargo, no es específico de asma ya que se puede encontrar elevado en bronquitis eosinofílica, rinitis alérgica, eczema y atopía. En pacientes con sospecha clínica de asma, sin tratamiento con corticoides inhalados, una FeNO elevada apoya el diagnóstico de asma eosinofílico y predice una buena respuesta al tratamiento con corticoides inhalados. La guía oficial de la American Thoracic Society (ATS) considera diagnóstico probable de asma eosinofílico una FeNO $>20$ partes por billón (ppb) en niños menores de 12 años y una FeNO > 25 ppb en mayores de 12 años $^{21}$. Se ha demostrado que los niños $<12$ años que tienen una FeNO $>35$ ppb $\mathrm{y}$ aquellos $>12$ años que tienen una FeNO $>50$ ppb tienen mayor probabilidad de tener diagnóstico de asma, crisis de asma y consultas en servicio de urgencia por esta causa ${ }^{22,23}$. La rápida caída de la FeNO frente al uso de corticoides 
inhalados es útil para monitorizar la adherencia al tratamiento de mantención y predice la pérdida del control del asma y recaída al suspender este tratamiento ${ }^{24-26}$. Es importante señalar que una FeNO normal no descarta el diagnóstico de asma bronquial ni contraindica un tratamiento con corticoides inhalados.

\section{Recuento de eosinófilos en sangre}

El aumento de eosinófilos en sangre es un marcador d atópica y no es específico de asma. Se ha observado que hay una asociación entre asma alérgico mediado por linfocitos $\mathrm{T}$ Helper 2 e inflamación eosinofílica de la vía aérea. En estos pacientes un recuento aumentado de eosinófilos se correlaciona con asma grave y se ha utilizado como biomarcador para terapia biológica $^{27-29}$. Un recuento de eosinófilos normal no descarta asma ni eosinofilia de la vía aérea ${ }^{30}$. Se considera eosinofilia un recuento absoluto mayor de 300 células $/ \mathrm{ml}^{31,32}$.

\section{$\operatorname{IgE}$ total}

Una IgE total elevada en un paciente asmático sugiere fenotipo alérgico. Sin embargo, no es específica de asma ya que puede estar elevada en infecciones virales, parasitosis, aspergilosis broncopulmonar alérgica, inmunodeficiencias, síndrome de Hiper IgE, dermatitis atópica y neoplasias entre otras. Por otro lado, una $\operatorname{IgE}$ total normal no descarta el diagnóstico de asma.

\section{c) Otros exámenes}

Niveles de vitamina D (25-hidroxivitamina D) en sangre

Se ha demostrado que un grupo de pacientes con asma bronquial pueden tener niveles bajos de vitamina $\mathrm{D}(<20 \mathrm{ng} / \mathrm{ml})$, lo que se asocia a función pulmonar disminuida, mayor frecuencia de crisis y menor respuesta al tratamiento con corticoides inhalados ${ }^{33,34}$. Se ha visto además que tener niveles adecuados de vitamina D mejora la biodisponibilidad de los corticoides, aumentando su eficiencia ${ }^{35}$. Así en pacientes con asma y niveles bajos de vitamina D se recomienda suplementarla ${ }^{36}$.

\section{Radiografía de tórax}

La radiografía de tórax anteroposterior y lateral está indicada en todo niño con sospecha de asma bronquial en su etapa diagnóstica. En la crisis de asma está indicada solo cuando existe sospecha clínica de complicaciones como neumonía, atelectasias o neumotórax.

\section{Clasificación del asma bronquial}

El asma bronquial debe clasificarse según la gravedad y de acuerdo al grado de control alcanzado con el tratamiento.

\section{a) Clasificación según la gravedad}

La gravedad de la enfermedad se define en base a la intensidad del tratamiento requerido para alcanzar un buen control de los síntomas y de las crisis de asma (Tabla 3$)^{5}$. La clasificación de la gravedad es dinámica en el tiempo, pudiendo cambiar en meses o años. Así, asma grave es aquel que requiere un tratamiento muy intenso para mantener un buen control o aquel que, a pesar de la intensidad del tratamiento, el control no es alcanzado. El asma grave a su vez se divide en asma grave de difícil control y asma grave resistente a tratamiento. El asma grave de difícil control es aquel que aparentemente no responde al tratamiento, pero con un manejo adecuado finalmente se logra controlar ${ }^{37}$. En esta categoría se incluye a pacientes con error en el diagnóstico; pacientes con comorbilidades que no están adecuadamente tratadas y que inciden negativamente en su evolución, siendo la rinitis alérgica una de las más frecuentes; deficiente adherencia al tratamiento de mantención; deficiente técnica inhalada y exposición a contaminantes ambientales o a alérgenos a los cuales el paciente esté sensibilizado. De todos estos factores por lejos el más frecuente es la deficiente adherencia al tratamiento de mantención ${ }^{38}$. Todos estos factores son potencialmente reversibles y cuando se logra corregirlos el asma finalmente se controla. Por otro lado, tenemos el asma grave resistente al tratamiento caracterizado por la ausencia de factores potencialmente modificables y en el cual, a pesar de un manejo óptimo con todo el tratamiento convencional disponible, no se logra el control ${ }^{37}$. Esta distinción es muy importante al momento de decidir escalar o no en la terapia, como veremos más adelante. Los pacientes con asma leve a moderado, deben tratarse en el nivel primario de atención y los pacientes con asma moderado no controlado y asma grave deben ser derivados a especialista.

Tabla 3. Clasificación de la gravedad del asma

\begin{tabular}{|ll|}
\hline Gravedad & Intensidad del tratamiento \\
\hline Leve & Nivel 1 y 2 de GINA \\
\hline Moderado & Nivel 3 de GINA \\
Severo & Nivel 4 y 5 de GINA \\
\hline
\end{tabular}




\section{b) Clasificación según el nivel de control}

El control del asma se refiere al grado de reducción o desaparición de los síntomas de asma debido al uso del tratamiento y refleja la efectividad de éste. Así tendremos asma bien controlado, parcialmente controlado y mal controlado (Tabla 4) ${ }^{5}$. En cada visita médica debe realizarse la evaluación del grado de control de la enfermedad, ya que de acuerdo a esto se efectúa el ajuste de la terapia. Una vez que se inicia el tratamiento, el manejo clínico y terapéutico de la enfermedad debe dirigirse a lograr y mantener el control. Así el grado de control determinará las decisiones sobre el tratamiento de mantención y el ajuste de dosis, según los pasos o escalones terapéuticos definidos por las guías de tratamiento ${ }^{5}$. Para la evaluación del control también existen cuestionarios validados, como el Test de Control del Asma (ACT). Existe una versión para niños de 4 a 11 años y otra para niños mayores de 12 años y adultos. De acuerdo con este cuestionario se considera asma bien controlado un puntaje $\geq 20$.

\section{Tratamiento}

El tratamiento del asma bronquial se basa en dos pilares: uno se refiere al tratamiento medicamentoso y el otro a la educación. Este debe incorporar las necesidades individuales de cada paciente, donde además de los fármacos utilizados es necesario entregar contenidos educativos, enseñar la técnica inhalatoria correcta e indicar un plan de acción escrito en caso de crisis. Además, el médico tratante debe identificar y tratar las comorbilidades ${ }^{39}$. Los objetivos principales del tratamiento son lograr un buen control de los síntomas y minimizar el riesgo de crisis, mejorando la calidad de vida del paciente y su familia y preservar la función pulmonar, con mínimos efectos adversos del tratamiento ${ }^{5,39,40}$.

\section{Medicamentos}

\section{Controladores}

Son fármacos que se utilizan en el tratamiento de mantención. Reducen la inflamación de la vía aérea, lo que permite el control de los síntomas y la disminución o desaparición de las crisis. El inicio del fármaco controlador debe realizarse inmediatamente después del diagnóstico y debe elegirse en forma individual para cada paciente considerando el grado de severidad, pero también valorar las preferencias del paciente con respecto al fármaco o modo de administración y los factores que puedan limitar su adherencia al tratamiento (Tabla 4) ) $^{5,40,41}$.

\section{a) Corticoides inhalados}

Son el tratamiento de elección para pacientes con asma bronquial pues se ha demostrado ampliamente que disminuyen la presencia de síntomas, reducen el riesgo de crisis, disminuyen la mortalidad, mejoran la calidad de vida y la función pulmonar $^{5,39}$. Los corticoides inhalados se pueden administrar mediante inhaladores presurizados o en polvo seco. Existen dispositivos que contienen solo corticoides y otros combinados con beta 2 agonistas de acción corta (salbutamol), beta 2 agonistas de acción larga (LABA: salmeterol y formoterol) y ultra larga (vilanterol) (Tabla 5). La adición de beta 2 agonistas de acción larga o ultra larga al corticoide inhalado aumenta la potencia de este último, mejorando la eficacia del tratamiento. Siempre se debe indicar la dosis más baja posible de corticoides inhalados que permita alcanzar el control de la enfermedad. Las dosis máximas administrables varían con la edad (Tabla 6). Cuando se utilicen inhaladores presurizados en escolares, éstos deben ser administrados con aerocámara con boquilla (ver más adelante sección educación).

\section{b) Antileucotrienos (Montelukast)}

Se ha demostrado ampliamente que estos medicamentos son menos efectivos como monotera-

Tabla 4. Evaluación del control de acuerdo a las guías GINA ${ }^{5}$

\begin{tabular}{|lccc|}
\hline Síntomas durante las últimas 4 semanas & $\begin{array}{c}\text { Bien } \\
\text { controlado }\end{array}$ & $\begin{array}{c}\text { Parcialmente } \\
\text { controlado }\end{array}$ & No controlado \\
\hline ¿Síntomas diurnos $>2$ veces/semana? & & & \\
¿Algún despertar nocturno debido al asma? & Ninguno de ellos & $1-2$ de ellos & $3-4$ de ellos \\
¿Necesidad de tratamiento de rescate $>2$ veces/semana? & & & \\
¿Alguna limitación de la actividad debida al asma? & & \\
\hline
\end{tabular}


Tabla 5. Corticoides inhalados disponibles en Chile

\begin{tabular}{|llll|}
\hline Medicamento & Presentación & Dosis & Restricción por edad \\
\hline Budesonida & Presurizado & $200 \mu \mathrm{g}$ & \\
\hline Budesonida & Polvo seco & $200 \mu \mathrm{g}$ & $>6$ años \\
\hline Fluticasona propionato & Presurizado & $50,125 \mathrm{y} 250 \mu \mathrm{g}$ & \\
\hline Fluticasona furoato & Polvo seco & $90 \mathrm{y} 182 \mu \mathrm{g}$ & $>12$ años \\
\hline Ciclesonida & Presurizado & $80 \mathrm{y} 160 \mu \mathrm{g}$ & $>6$ años \\
\hline Budesonida/formoterol & Presurizado & $80 / 4,5 \mathrm{y} 160 / 4,5 \mu \mathrm{g}$ & $>4$ años \\
\hline Budesonida/formoterol & Polvo seco & $160 / 4,5$ y $320 / 9 \mu \mathrm{g}$ & $>6$ años \\
\hline Fluticasona propionato/salmeterol & Presurizado & $50 / 25,125 / 25$ y $250 / 25 \mu \mathrm{g}$ & $>4$ años \\
\hline Fluticasona propionato/salmeterol & Polvo seco & $100 / 50,250 / 50$ y $500 / 50 \mu \mathrm{g}$ & $>6$ años \\
\hline Fluticasona furoato/vilanterol & Polvo seco & $92 / 22$ y $184 / 22 \mu \mathrm{g}$ & $>12$ años \\
\hline Beclometasona/salbutamol & Presurizado & $50 / 100 \mu \mathrm{g}$ & \\
\hline
\end{tabular}

Tabla 6. Dosis baja, media y alta de corticoides inhalados según la edad

\begin{tabular}{|lcccccc|}
\hline & \multicolumn{3}{c}{$\begin{array}{c}\text { Niños/as de 6 a 11 años } \\
\text { (Dosis diaria en } \boldsymbol{\mu g} / \mathbf{d i ́ a )}\end{array}$} & \multicolumn{3}{c|}{$\begin{array}{c}\text { Niños/as }>\text { 12 años } \\
\text { (Dosis diaria en } \boldsymbol{\mu g} / \mathbf{d i ́ a )}\end{array}$} \\
Medicamento & Dosis baja & Dosis media & Dosis alta & Dosis baja & Dosis media & Dosis alta \\
Budesonida & $100-200$ & $>200-400$ & $>400$ & $200-400$ & $>400-800$ & $>800$ \\
Fluticasona propionato & $100-200$ & $>200-500$ & $>500$ & $100-250$ & $>250-500$ & $>500$ \\
Fluticasona furoato & No aplica & No aplica & No aplica & 100 & No aplica & 200 \\
Ciclesonida & 80 & $>80-160$ & $>160$ & $80-160$ & $>160-320$ & $>320$ \\
\hline
\end{tabular}

pia que los corticoides inhalados para el control del asma ${ }^{5}$. Podrían ser adecuados para iniciar un tratamiento controlador en algunos casos muy seleccionados, no graves y cuando el paciente o sus cuidadores rehúsan usar corticoides inhalados ${ }^{5}$. Pueden ser usados como tratamiento adicional al uso de corticoides inhalados para aumentar la intensidad del tratamiento, aunque esta mezcla es menos efectiva que la combinación de corticoides inhalados con beta 2 agonistas de acción larga o ultra larga ${ }^{42}$. Es importante además tener en consideración los efectos adversos que pueden ocurrir en hasta un $10 \%$ de los casos. Estos se observan habitualmente luego de una semana de iniciado el tratamiento y pueden consistir en irritabilidad, agresividad, trastornos del sueño, pesadillas y efectos neuropsiquiátricos como depresión e ideación suicida, sobretodo en adolescentes. Es por este motivo que en marzo de 2020 la Administración de Alimentos y Medicamentos (FDA) de los Estados Unidos determinó la necesidad de instalar un recuadro de advertencia en los envases de estos medicamentos.

\section{c) Anticolinérgicos de acción prolongada (tiotropio)}

El tiotropio es un antimuscarínico de acción prolongada el cual puede ser usado como terapia de adición en pacientes de 6 años o más. Se ha demostrado que sumado al tratamiento con corticoides inhalados puede mejorar la función pulmonar y reducir las crisis de asma ${ }^{5,6,43}$. Este medicamento se administra mediante un dispositivo llamado Respimat que produce una nube de vapor. Se administra directamente en la boca o mediante aerocámara dependiendo de la capacidad de coordinación del paciente. La dosis es de $5 \mu \mathrm{g} 1 \mathrm{vez}$ al día, dosis que se obtiene aplicando 2 inhalaciones.

\section{Medicamentos de rescate}

Por muchos años se consideró al salbutamol como el medicamento de rescate de elección. Sin embargo, a partir del año 2019 las guías GINA establecen que el uso de salbutamol solo ya no es recomendado como terapia de rescate 
en adolescentes y adultos por ser poco seguro. En todo paciente se recomienda el uso de broncodilatadores siempre asociados a un corticoide inhalado, especialmente en aquellos pacientes que no están recibiendo tratamiento de mantención ${ }^{5}$. Este grupo de pacientes corresponde a los catalogados como leves o aquellos con deficiente adherencia al tratamiento de mantención. El fundamento de este cambio de paradigma se debe a que un grupo significativo de los pacientes clasificados como leves, como aquellos con deficiente adherencia al tratamiento, pueden presentar crisis graves, incluso con resultado fatal. Esto se debería a una inflamación mantenida y progresiva de la vía aérea, especialmente por un exceso de confianza en el uso de salbutamol como monoterapia en forma intermitente, dado el rápido alivio de los síntomas que este medicamento produce. Diversos estudios demuestran que el uso de salbutamol como monoterapia aumenta el riesgo de morir por asma y se asocia además a un mayor número de consultas a servicios de urgencia ${ }^{44-46}$. Por otro lado, se ha demostrado que dosis bajas de corticoides inhalados son altamente efectivas para reducir los síntomas de asma, el riesgo de hospitalización y de morir por esta enfermedad ${ }^{47,48}$. Este cambio en el tratamiento recomendado por las nuevas guías GINA proviene de la evidencia aportada por la estrategia SMART (single maintenance and reliever therapy), que consiste en el uso de la asociación de un corticoide inhalado (budesonida) más un beta 2 agonista de acción prolongada (formoterol) tanto para mantención como para rescate. formoterol, además de ser un beta 2 agonista de acción prolongada, tiene un rápido inicio de acción, por lo que puede ser utilizado para rescate, no así salmeterol o vilanterol. Un metaanálisis que evaluó la terapia SMART en 22.524 pacientes utilizando la mezcla budesonida/formoterol, demostró una reducción del riesgo de crisis de asma comparado con el uso de un corticoide inhalado de mantención más el uso de salbutamol como terapia de rescate ${ }^{49}$. Por otro lado, el estudio Sygma 1 evidenció que, en pacientes mayores de 12 años con asma leve, el uso a demanda de budesonida/formoterol redujo significativamente las crisis de asma y el tiempo transcurrido a la primera crisis severa en comparación con el uso de salbutamol solo. En el estudio Sygma 2 se observó además que esta mezcla no era inferior al uso regular de corticoides inhalados más salbutamol a demanda, en cuanto a la frecuencia anual de crisis y su severidad, con una dosis de corticoides inhalados incluso menor ${ }^{50,51}$.

\section{Otros medicamentos}

\section{a) Terapias biológicas}

Las terapias biológicas están dirigidas a pacientes con asma grave resistente a tratamiento, con fenotipo eosinofílico alérgico y no alérgico. Están reservadas para ser administradas solo en el nivel terciario por especialistas. Los anticuerpos monoclonales disponibles en Chile son omalizumab y mepolizumab. Próximamente también habrá disponibilidad en nuestro país de dupilumab (bloqueador del receptor de IL4 e IL13) y benralizumab (bloqueador del receptor de IL5).

\section{Omalizumab}

Anticuerpo monoclonal humanizado anti IgE. Su uso está aprobado para pacientes a partir de los de 6 años. Es la terapia biológica de primera elección en niños ya que hay múltiples estudios en la población pediátrica, desde hace más de una década, que avalan su seguridad y eficacia. Ejerce su acción bloqueando la IgE libre circulante, evitando que ésta se una a su receptor de alta afinidad sobre mastocitos y así logra inhibir la cascada alérgica. Además, reduce la expresión de receptores de IgE presentes sobre células presentadoras de antígeno, lo que disminuye la sensibilización alérgica. Diferentes metaanálisis demuestran que omalizumab es seguro en niños, reduce el riesgo de crisis de asma, consultas a servicios de urgencia y hospitalizaciones, permitiendo que los pacientes puedan incluso bajar el nivel del tratamiento controlador y mejorar significativamente su calidad de vida ${ }^{41,52}$. Cuando el tratamiento es exitoso los pacientes logran retomar las actividades deportivas y de la vida diaria que habían suspendido por la gravedad de la enfermedad ${ }^{53}$. Los requisitos para su uso son tener asma grave resistente a tratamiento, test cutáneo $(+)$ e $\mathrm{IgE}$ sobre $30 \mathrm{mg} / \mathrm{dl}$. La dosis se calcula en base al peso y nivel de IgE que tenga el paciente. Su administración es subcutánea en forma mensual o bisemanal ${ }^{53}$. En general se hace una prueba terapéutica por 4 meses. Si al cabo de esta prueba el paciente mejora clínicamente la terapia se mantiene, de lo contrario debe suspenderse.

\section{Mepolizumab}

Anticuerpo monoclonal humanizado anti interleucina 5 (IL5). Su uso ha sido aprobado recientemente para niños desde los 6 años. La IL5 es fundamental en la maduración y liberación de los eosinófilos desde la médula ósea hacia la sangre y es un potente quimioatrayente de estas 
células hacia la mucosa respiratoria, disminuyendo además su apoptosis. Los eosinófilos son los responsables de la respuesta alérgica tardía, manteniendo la inflamación en la vía aérea. Mepolizumab actúa contrarrestando la acción de los eosinófilos a nivel bronquial al unirse a la IL5 circulante, impidiendo que ésta se una a su receptor. La acción de mepolizumab se ve reflejada en un menor recuento de eosinófilos en sangre y esputo y en una disminución significativa de las crisis de asma, de las hospitalizaciones y de las consultas a servicios de urgencia, con una mejoría asociada de la calidad de vida ${ }^{41}$. Su uso está indicado en pacientes con asma grave resistente a tratamiento con recuento de eosinófilos actual mayor a 150 células $/ \mathrm{ml}$ o histórico mayor de 300 células $/ \mathrm{ml}^{41,52}$. Es muy importante recalcar que mepolizumab ha demostrado ser eficaz principalmente en el asma eosinofílico no alérgico del adulto. A diferencia de omalizumab existe poca evidencia científica de su eficacia en niños, sobretodo en menores de 12 años. Su uso en la población pediátrica pudiera estar indicado en casos de asma grave resistente a tratamiento en el que el uso previo de omalizumab haya fracasado.

\section{b) Vitamina $D$}

Existe evidencia que muestra una relación entre niveles bajos de vitamina D y crisis frecuentes y respuesta reducida a corticoides. Algunos estudios demuestran que la suplementación de vitamina $\mathrm{D}$ podría reducir el riesgo de crisis de asma. Sin embargo, esta evidencia es débil y no se ha demostrado que mejore el control de la enfermedad. Siendo el asma una enfermedad crónica, se recomienda mantener niveles plasmáticos de vitamina D sobre $30(\mathrm{ng} / \mathrm{ml})^{5,54}$.

\section{Esquema de tratamiento por niveles}

El tipo de tratamiento a indicar va a depender del nivel de síntomas que presente inicialmente el paciente y de su edad (Tabla 7). Hasta aquí se han expuesto las distintas alternativas de tratamiento disponibles en nuestro país, de acuerdo con lo que propone la guía GINA. Esta más que una guía es una estrategia, la cual puede ser tomada y adaptada a la realidad de cada país, de acuerdo a los medicamentos disponibles. Luego de analizar toda la evidencia científica y de acuerdo al arsenal terapéutico disponible en nuestro país a continuación detallamos las recomendaciones de tratamiento que propone la Comisión de Asma SOCHINEP-SER para el manejo del asma en el escolar (Tablas 8 y 9).

\section{Revisión de la respuesta al tratamiento}

Una vez iniciado el tratamiento, el paciente debe ser controlado al mes. En esta visita es importante evaluar el grado de control de la enfermedad, la adherencia a la terapia, la técnica inhalatoria y reforzar contenidos educativos (ver sección educación). Respecto del control del asma, se debe determinar si los síntomas están presentes, si han disminuido o si han sido eliminados a causa del tratamiento. La estrategia para lograr un buen control de la enfermedad implica un ciclo continuo que incluye evaluación del paciente (diagnóstico, control de los síntomas, factores de riesgo, técnica inhalatoria, adherencia al tratamiento y preferencias del paciente), ajuste de la terapia (medicamentos, medidas no farmacológicas y tratamiento de los factores de riesgo modificables) y revisión de la respuesta (síntomas, crisis de asma, efectos colaterales, satisfacción del paciente y función pulmonar). Este ciclo debe repetirse en cada control mé-

Tabla 7. Nivel de tratamiento del asma según GINA, de acuerdo a los síntomas iniciales 5

\begin{tabular}{|ll|} 
& Características \\
Nivel 1 & $\begin{array}{l}\text { Síntomas }<2 \text { veces al mes } \\
\text { Sin factores de riesgo de crisis* }\end{array}$ \\
Nivel 2 & $\begin{array}{l}\text { Síntomas de asma o necesidad de tratamiento de rescate } 2 \text { o más veces al mes y/o factores de riesgo de } \\
\text { crisis }\end{array}$ \\
Nivel 3 & $\begin{array}{l}\text { Síntomas de asma más de } 2 \text { veces a la semana o diarios } \\
\text { Despertares nocturnos } 1 \text { vez a la semana o más } \\
\text { Con factores de riesgo de crisis* }\end{array}$ \\
Nivel 4 & Presentación inicial con asma grave de difícil control o con crisis de asma moderada u hospitalizada \\
\hline
\end{tabular}

*Factores de riesgo de crisis son: alta frecuencia de uso de salbutamol, comorbilidades (rinitis alérgica, obesidad, reflujo gastroesofágico, alergia alimentaria entre otros), exposición a contaminantes o alérgenos, problemas psicológicos o socioeconómicos, $\mathrm{VEF}_{1}$ bajo, respuesta broncodilatadora positiva, antecedente de intubación o de alguna hospitalización en Unidad de Paciente Crítico, 1 o más crisis graves en los últimos 12 meses. 
Tabla 8. Tratamiento de pacientes de 12 años o más

\begin{tabular}{|c|c|}
\hline & Medicamentos \\
\hline Nivel 1 & $\begin{array}{l}\text { - Dosis baja de Budesonida/Formoterol a demanda * (se recomienda utilizar mezcla con la menor } \\
\text { dosis de Budesonida posible) o } \\
\text { - Beclometasona/Salbutamol a demanda o } \\
\text { - Uso de Budesonida o Fluticasona toda vez que se utilice salbutamol }\end{array}$ \\
\hline Nivel 2 & $\begin{array}{l}\text { - Dosis bajas de corticoides inhalados de uso permanente o } \\
\text { - Budesonida/Formoterol a demanda* o } \\
\text { - Montelukast de uso permanente (considerar inferioridad terapéutica en comparación con corticoides } \\
\text { inhalados y los posibles efectos adversos) } \\
\\
\text { - Rescate con Salbutamol en caso de usar Fluticasona o Budesonida } \\
\text { - Rescate con Budesonida/Formoterol* o Beclometasona/Salbutamol o Budesonida o Fluticasona toda } \\
\text { vez que se utilice salbutamol, en caso de usar Montelukast permanente }\end{array}$ \\
\hline Nivel 3 & $\begin{array}{l}\text { - Dosis bajas de corticoides inhalados más LABA a permanencia } \\
\text { o dosis media de corticoides inhalados a permanencia o } \\
\text { - Dosis bajas de corticoides inhalados a permanencia más Montelukast a permanencia } \\
\text { - Rescate con Salbutamol en caso de usar: Fluticasona/Salmeterol, Fluticasona/Vilanterol, Fluticasona } \\
\text { o Budesonida } \\
\text { - Rescate con Budesonida/Formoterol* en caso de utilizar esta misma mezcla de mantención }\end{array}$ \\
\hline Nivel 4 & $\begin{array}{l}\text { REFERIR A ESPECIALISTA } \\
\text { - Dosis media de corticoides inhalados más LABA a permanencia o } \\
\text { - Dosis alta de corticoides inhalados a permanencia más Tiotropio o Montelukast } \\
\text { - Rescate con Salbutamol en caso de usar Fluticasona/Salmeterol, Fluticasona/Vilanterol, Fluticasona o } \\
\text { Budesonida } \\
\text { - Rescate con Budesonida/Formoterol* en caso de utilizar esta misma mezcla de mantención }\end{array}$ \\
\hline Nivel 5 & $\begin{array}{l}\text { REFERIR A ESPECIALISTA } \\
\text { - Dosis alta de corticoides inhalados más LABA a permanencia. } \\
\text { Agregar: Tiotropio (si no lo está usando) y/u Omalizumab o Mepolizumab } \\
\text { Considerar corticoides orales si no hay disponibilidad de otras terapias } \\
\text { - Rescate con Salbutamol en caso de usar Fluticasona/Salmeterol o Fluticasona/Vilanterol } \\
\text { - Rescate con Budesonida/Formoterol* en caso de utilizar esta misma mezcla de mantención }\end{array}$ \\
\hline
\end{tabular}

En negrita se indica tratamiento de elección y con letras normales alternativas de segunda opción. *Máximo 8 inhalaciones al día.

$\operatorname{dico}^{5,41}$. Posterior al primer control, las visitas deben realizarse cada 3 meses si el paciente está estable, de lo contrario deben ser mensuales o incluso quincenales ${ }^{5,55}$. Como ya se mencionó si el paciente no ha alcanzado el control de la enfermedad en el nivel de tratamiento en el que está, antes de pensar aumentar la intensidad del tratamiento se deben descartar factores potencialmente reversibles que permitan mejorar el control sin tener que escalar innecesariamente en la terapia (ver sección educación). Por otro lado, el descenso en la intensidad del tratamiento se indica cuando se alcanza el control y éste es mantenido por al menos tres meses, con pruebas de función pulmonar normales $5,39,41,56$. En estos casos se recomienda el descenso de $50 \%$ de la dosis administrada previamente, manteniendo un control estrecho del paciente.

\section{Tratamiento de la crisis de asma}

\section{Definición}

Deterioro agudo o subagudo de los síntomas y de la función pulmonar respecto del estado habitual del paciente, con presencia de obstrucción bronquial, que se manifiesta por disnea progresiva, tos, sibilancias, sensación de opresión torácica o una combinación de estos síntomas ${ }^{5}$. También puede ser el debut o la primera manifestación de la enfermedad. En los casos más graves se presentan signos propios de insuficiencia respiratoria ${ }^{17}$. La palabra exacerbación no se debería utilizar más para definir esta condición ya que implica que el evento es trivial y recuperable ${ }^{57}$. El término correcto y que utilizaremos de ahora en adelante será "crisis" y lo preferimos porque lleva implícito el concepto de gravedad y de 
Tabla 9. Tratamiento de pacientes de 5 a 11 años

\begin{tabular}{|c|c|}
\hline & Medicamentos \\
\hline Nivel 1 & $\begin{array}{l}\text { - Dosis baja de Budesonida/Formoterol a demanda* (se recomienda utilizar mezcla con la menor } \\
\text { dosis de Budesonida posible) o } \\
\text { - Beclometasona/Salbutamol a demanda o } \\
\text { - Uso de Budesonida o Fluticasona toda vez que se utilice salbutamol }\end{array}$ \\
\hline Nivel 2 & $\begin{array}{l}\text { - Dosis bajas de corticoides inhalados de uso permanente o } \\
\text { - Montelukast de uso permanente (considerar inferioridad terapéutica en comparación con corticoides } \\
\text { inhalados y los posibles efectos adversos) } \\
\text { - Rescate con Salbutamol en caso de usar Fluticasona o Budesonida } \\
\text { - Rescate con Budesonida/Formoterol* o Beclometasona/Salbutamol o Budesonida o Fluticasona toda } \\
\text { vez que se utilice salbutamol, en caso de usar Montelukast permanente }\end{array}$ \\
\hline Nivel 3 & $\begin{array}{l}\text { - Dosis bajas de corticoides inhalados más LABA a permanencia o dosis media de corticoides } \\
\text { inhalados a permanencia o } \\
\text { - Dosis bajas de corticoides inhalados a permanencia más Montelukast a permanencia } \\
\\
\text { - Rescate con Salbutamol en caso de usar Fluticasona/Salmeterol, Fluticasona o Budesonida } \\
\text { - Rescate con Budesonida/Formoterol* en caso de utilizar esta misma mezcla de mantención }\end{array}$ \\
\hline Nivel 4 & $\begin{array}{l}\text { REFERIR A ESPECIALISTA } \\
\text { - Dosis media de corticoides inhalados más LABA a permanencia } \\
\text { - Se puede agregar Tiotropio o Montelukast } \\
\\
\text { - Rescate con Salbutamol en caso de usar Fluticasona/Salmeterol } \\
\text { - Rescate con Budesonica/Formoterol* en caso de utilizar esta misma mezcla de mantención }\end{array}$ \\
\hline Nivel 5 & $\begin{array}{l}\text { REFERIR A ESPECIALISTA } \\
\text { - Dosis alta de corticoides inhalados más LABA a permanencia } \\
\text { Agregar: Tiotropio (si no lo está usando) y/o Omalizumab o Mepolizumab } \\
\text { Considerar corticoides orales, si no hay disponibilidad de otras terapias } \\
\text { - Rescate con Salbutamol en caso de usar Fluticasona/Salmeterol } \\
\text { - Rescate con Budesonica/Formoterol* en caso de utilizar esta misma mezcla de mantención }\end{array}$ \\
\hline
\end{tabular}

En negrita se indica tratamiento de elección y con letras normales alternativas de segunda opción. *Máximo 8 inhalaciones al día.

mayor riesgo. Por todo lo que implican las crisis de asma estas deben constituirse como banderas rojas, siendo uno de los objetivos del tratamiento tener tolerancia cero con ellas ${ }^{58,59}$.

\section{Factores desencadenantes de crisis de asma}

Las infecciones respiratorias, son la causa más común de crisis de asma a toda edad ${ }^{60}$. El rinovirus es el agente infeccioso más comúnmente encontrado, especialmente el rinovirus $\mathrm{C}$, siendo el que más se asocia a crisis de asma ${ }^{61-63}$. Se ha visto además que ciertos polimorfismos del receptor del rinovirus $\mathrm{C}$ (CDHR3) se asocian con crisis severas en niños ${ }^{64,65}$. El rol de las infecciones bacterianas en episodios agudos de asma en escolares es menos claro que con los virus, aunque se han detectado Moraxella catarrhalis, Streptococcus pneumoniae y Heaemophillus influenzae ${ }^{66}$. La exposición ambiental a alérgenos, contaminación y humo del tabaco también son gatillantes fre- cuentes de crisis de asma ${ }^{67,68}$. Las crisis de asma presentan un patrón de variación estacional, con un alza en otoño probablemente relacionada con infección por rinovirus y con la suspensión del tratamiento controlador durante los meses previos (regreso del verano) y otra en primavera asociada a exposición a pólenes ${ }^{3,69,70}$.

\section{Evaluación de la gravedad de la crisis de asma}

El primer paso en la evaluación de la gravedad de una crisis de asma es realizar una anamnesis completa y dentro de esta evaluación es muy importante determinar si el paciente tiene factores de riesgo para presentar una crisis severa y de fallecer por esta causa ${ }^{59}$ (Tabla 10). Para evaluar la intensidad y gravedad de la crisis de asma, es fundamental tener una valoración objetiva del examen físico. Existen diferentes índices o puntajes. Aunque ninguno de ellos ha sido validado en niños, el Pulmonary Score o puntaje de asma 
Tabla 10. Factores que aumentan el riesgo de morir por crisis de asma

\begin{tabular}{|l|}
\hline Antecedente de intubación y ventilación mecánica \\
Hospitalización y visita a servicio urgencia por asma en los últimos 12 meses \\
No estar recibiendo tratamiento permanente con corticoides inhalados \\
Sobreuso de salbutamol, especialmente más de un canister por mes \\
Historia de problemas psiquiátricos o psicosociales \\
Poca adherencia al tratamiento de mantención o falta de un plan de acción escrito \\
Alergia alimentaria
\end{tabular}

Tabla 11. Valoración clínica de la crisis. Pulmonary Score ${ }^{71,72}$

\begin{tabular}{|cccll|}
\hline Puntaje & \multicolumn{2}{c}{$\begin{array}{l}\text { Frecuencia respiratoria } \\
<\mathbf{6} \text { años }\end{array}$} & Sibilancias & $\begin{array}{l}\text { Uso de musculatura accesoria } \\
\text { (Esternocleidomastoideo ECM) }\end{array}$ \\
\hline 0 & $<30$ & $<20$ & No & No \\
\hline 1 & $31-45$ & $21-35$ & Final de la espiración & Incremento leve \\
\hline 2 & $46-60$ & $36-50$ & En toda la espiración & Aumentado \\
3 & $>60$ & $>50$ & $\begin{array}{l}\text { En inspiración y espiración sin } \\
\text { fonendoscopio* }\end{array}$ & Actividad máxima \\
\hline
\end{tabular}

*Si no hay sibilancias y la actividad del ECM está aumentada, poner 3 puntos en ítem sibilancias. Puntuación 0-3 en cada uno de los ítems (mínimo 0 y máximo 9). Crisis leve: 0-3 puntos; moderada: 4-6 puntos; grave: 7-9 puntos.

agudo, ha sido ampliamente usado y permite tomar decisiones en el tratamiento de la crisis ${ }^{71,72}$ (Tabla 11).

\section{Oximetría de pulso}

Es un complemento fundamental del examen físico, ya que detecta la presencia de hipoxemia que puede pasar desapercibida al examen físico y permite establecer también la severidad de la crisis de asma, junto con el puntaje de asma agudo (Tabla 12).

\section{Espirometría o flujo espiratorio máximo (PEF)}

En la medida de lo posible se recomienda objetivar el grado de obstrucción bronquial al momento de la crisis ya que esto se correlaciona bien con la gravedad del asma ${ }^{73,74}$. Para esto se puede utilizar un flujómetro (FEM) o un espirómetro $\left(\mathrm{VEF}_{1}\right)($ Tabla 13).

\section{Gases arteriales}

Se deben solicitar en episodios graves que no respondan a la terapia, en el contexto de un paciente hospitalizado. Una $\mathrm{PaCO}_{2}$ normal o elevada es indicador de empeoramiento de la crisis de asma ya que refleja fatiga de los músculos respiratorios lo que conduce a una insuficiencia respiratoria aguda hipercápnica inminente.
Tabla 12. Valoración global de la gravedad de la crisis integrando el Pulmonary Score y la saturación de oxígeno

\begin{tabular}{|lcc|}
\hline & $\begin{array}{c}\text { Pulmonary } \\
\text { score }\end{array}$ & $\begin{array}{c}\text { Saturación } \\
\text { de oxígeno* }\end{array}$ \\
\hline Leve & $0-3$ & $94 \%$ \\
Moderada & $4-6$ & $91-93 \%$ \\
Grave & $7-9$ & $<91 \%$ \\
\hline
\end{tabular}

* En caso de discordancia entre la puntuación clínica y la saturación de oxígeno, se utilizará el de mayor gravedad.

Tabla 13. Monitoreo funcional de gravedad del asma

\begin{tabular}{|llcl|}
\hline Índice & Leve & Moderada & Grave \\
$\mathrm{PEF}$ & $>70 \%$ & $40-69 \%$ & $<40 \%$ \\
$\mathrm{PaO}_{2}$ & Normal & $>60 \mathrm{mmHg}$ & $\begin{array}{l}<60 \mathrm{mmHg} \\
\text { posible } \\
\text { cianosis }\end{array}$ \\
$\mathrm{PCO}_{2}$ & $<42 \mathrm{mmHg}$ & $<42 \mathrm{mmHg}$ & $\begin{array}{l}>42 \mathrm{mmHg} \\
\text { posible falla } \\
\text { respiratoria }\end{array}$ \\
\hline $\mathrm{SaO}_{2}$ & $>94 \%$ & $91-94 \%$ & $<91 \%$ \\
\hline
\end{tabular}

$\mathrm{PEF}=$ Flujo espiratorio cúspide $\mathrm{SaO}_{2}=$ saturación de oxígeno. 


\section{Radiografía de tórax}

No se aconseja solicitarla de rutina ya que no modifica el tratamiento de la crisis de asma, a menos que se sospeche una complicación aguda como neumonía, atelectasia o neumotórax ${ }^{75}$.

\section{Tratamiento farmacológico de la crisis asmática}

El objetivo del tratamiento de una crisis de asma es reducir el riesgo de compromiso vital del paciente, revertir la hipoxemia y la obstrucción al flujo aéreo lo más rápidamente posible (Figura 1).

\section{1) Tratamiento de primera línea}

\section{Oxigenoterapia}

La primera prioridad en el manejo de una crisis asmática debe ser el tratamiento de la hipoxemia, tomando en cuenta la oximetría de pulso y también el grado de dificultad respiratoria del paciente. El objetivo es lograr una saturación de oxígeno $\geq 94 \%$, administrando oxígeno $\left(\mathrm{O}_{2}\right)$ humidificado, a través de una cánula nasal o de una mascarilla de Venturi ${ }^{5}$. Cuando los requerimientos de $\mathrm{O}_{2}$ son elevados $\left(\mathrm{FiO}_{2}>0,5\right)$ es preferible administrar $\mathrm{O}_{2}$ con mascarilla y reservorio. $\mathrm{Si}$ el paciente mantiene requerimientos de $\mathrm{O}_{2}$ elevados o presenta dificultad respiratoria, a pesar del manejo farmacológico que se detallará a continuación, debe considerarse su hospitalización.

\section{Beta 2 agonistas de acción corta}

El Salbutamol constituye la primera línea de tratamiento de una crisis asmática a toda edad, prefiriéndose la vía inhalatoria. En cuanto al modo de su administración por vía inhalada se ha demostrado que la nebulización no tiene ventajas sobre los inhaladores de dosis medida (IDM) ${ }^{76,77}$. La dosis depende de la gravedad de la crisis. En crisis leves se indica salbutamol 2-4 puffs cada 20 min por 3 veces y en crisis moderadas 4-8 puffs cada 20 min por 3 veces. Cuando el pacien-

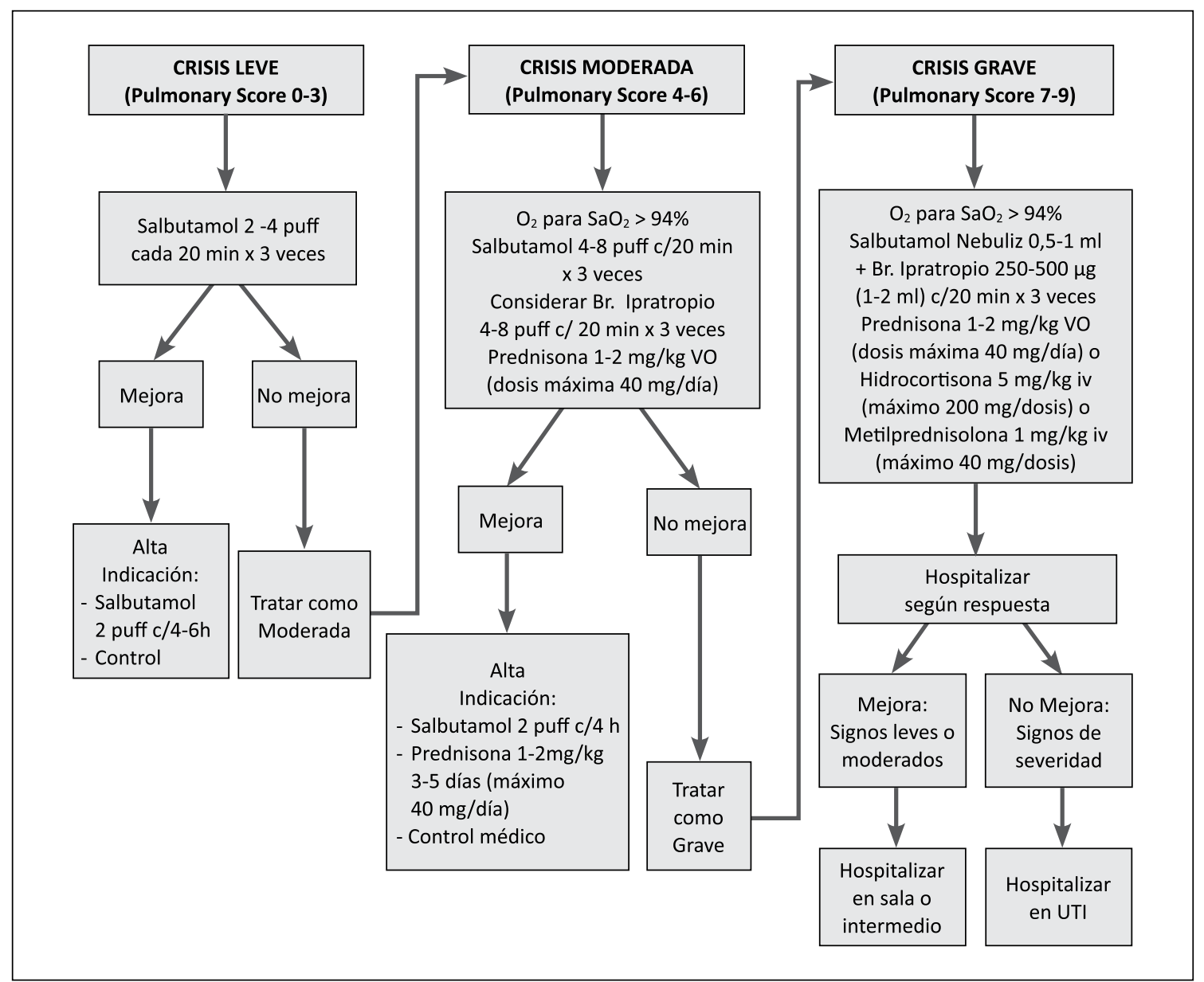

Figura 1. Diagrama de flujo del manejo de la crisis asmática. Los casos leves, que presenten factores de riesgo (Tabla 10) deben tratarse como moderados. $\min =$ minutos; $\mathrm{h}=$ hora; $\mathrm{SaO}_{2}=$ saturación de Oxígeno; Br. Ipratropio = Bromuro de Ipratropio; Nebuliz $=$ Nebulización; iv $=$ intravenoso. 
te mejora se puede continuar con 2-4 puffs cada $4 \mathrm{~h}$. En las crisis severas se puede administrar salbutamol mediante nebulización con $\mathrm{O}_{2}$ a un flujo de $7 \mathrm{l} / \mathrm{min}$. La dosis de salbutamol para nebulización es de 0,5 a $1 \mathrm{ml}(2,5 \mathrm{a} 5 \mathrm{mg})$ más suero fisiológico hasta alcanzar un volumen de $4 \mathrm{ml}$ y se repite cada 20 min por 3 veces. Si se observa mejoría del paciente, continuar con inhalador de dosis medida y aerocámara. El uso de salbutamol en nebulización continua no se asocia a un mejor resultado al compararlo con la administración frecuente e intermitente de salbutamol ${ }^{78}$.

\section{Bromuro de ipratropio}

$\mathrm{Su}$ uso precoz junto con salbutamol ha demostrado tener un efecto sinérgico, mejorando la función pulmonar y reduciendo el riesgo de hospitalización al compararlo con salbutamol solo $^{79}$. Su uso está indicado en las crisis severas y en las moderadas que no responden a la terapia con salbutamol durante la primera hora de manejo. La dosis de bromuro de Ipratropio para nebulización es de 250 a $500 \mu \mathrm{g}(1-2 \mathrm{ml})$. Se debe administrar junto con salbutamol cada 20 a $30 \mathrm{~min}$ por 3 veces, durante la primera hora. Luego debe reducirse su frecuencia a cada 4 a $6 \mathrm{~h}$ o suspenderse. Este fármaco como monoterapia, no está recomendado para ser utilizado en el manejo inicial de la crisis de asma y no se ha demostrado su utilidad en el paciente hospitalizado ya que no logra reducir los días de hospitalización ni mejorar otros parámetros ${ }^{80,81}$.

\section{Corticoides sistémicos}

Están indicados en el manejo de las crisis de asma moderadas y severas. Su uso precoz reduce el riesgo de hospitalización y de recaídas ${ }^{82}$. Se ha demostrado que el uso de prednisona oral en dosis de 1 a $2 \mathrm{mg} / \mathrm{kg} /$ día (máximo $40 \mathrm{mg} /$ día) en una dosis por 3 a 5 días, es igualmente efectivo que el uso de hidrocortisona endovenosa en dosis de $5 \mathrm{mg} / \mathrm{kg} /$ dosis (máximo $200 \mathrm{mg}$ ) cada 6 h y que el de metilprednisolona endovenosa en dosis de $1 \mathrm{mg} / \mathrm{kg} /$ dosis (máximo $40 \mathrm{mg}$ ) cada $6 \mathrm{~h}^{83}$. Siempre debe preferirse la vía oral, a menos que el paciente esté grave o presente vómitos o dificultad para deglutir. El uso de dosis altas de corticoides inhalados para la crisis de asma no está recomendado ${ }^{84}$.

\section{2) Tratamiento de segunda línea}

\section{Sulfato de magnesio}

Se utiliza en el servicio de urgencia, en pacientes con crisis de asma severas o que cursan con $\mathrm{VEF}_{1}<60 \%$ y solo si después de $1 \mathrm{~h}$ de terapia estándar no hay una respuesta satisfactoria ${ }^{85}$. $\mathrm{Su}$ uso ha demostrado reducir la necesidad de ventilación mecánica y de hospitalización, pero no se ha observado beneficio en pacientes leves o moderados ${ }^{86,87}$. Se inicia su administración con un bolo de 50 a $75 \mathrm{mg} / \mathrm{kg}$ y luego se continúa con una infusión continua a razón de $40 \mathrm{mg} / \mathrm{kg} /$ hora por $4 \mathrm{~h}$. Si bien algunos estudios avalan su administración en forma nebulizada, la evidencia global al respecto es débil, por lo que no se recomienda ${ }^{88}$.

\section{Ventilación mecánica no invasiva (VNI)}

La VNI es un método de apoyo ventilatorio con presión positiva, que se entrega a través de una interfase (mascarilla nasal o facial). La principal ventaja de esta forma de ventilación es que permite evitar las complicaciones que presentan los pacientes asmáticos sometidos a intubación y ventilación mecánica invasiva o convencional ${ }^{89}$.

\section{3) Otras terapias farmacológicas}

\section{Epinefrina}

Sólo está indicada en crisis asmáticas asociadas a anafilaxia o angioedema. Se utiliza una dosis intramuscular de $0,3 \mathrm{mg}(0,3 \mathrm{ml}$ de adrenalina acuosa $1: 1.000)$ para niños de 6-12 años la cual de ser necesario puede repetirse hasta 3 veces cada $15 \mathrm{~min}^{90}$. En adolescentes y adultos la dosis es de $0,5 \mathrm{mg}(0,5 \mathrm{ml}$ de adrenalina acuosa $1: 1000)$.

\section{Metilxantinas}

El uso de aminofilina endovenosa asociada a beta 2 agonistas de acción corta y corticoides sistémicos en niños con crisis graves, mejora la función pulmonar a las $6 \mathrm{~h}$ de su administración, pero no reduce los síntomas ni la duración de la hospitalización. Debido a su estrecho margen terapéutico, la necesidad de monitorizar sus niveles plasmáticos y sus potenciales efectos adversos, no se recomienda su uso de rutina en el manejo de las crisis de asma severas ${ }^{91}$.

\section{Heliox}

El uso de esta mezcla de helio y oxígeno disminuye el trabajo respiratorio y mejora la entrega y depósito de partículas inhaladas, como beta 2 agonistas de acción corta en la vía aérea distal. Sin embargo, puede ser insuficiente para mejorar la oxigenación en pacientes con hipoxemia severa. Su uso rutinario como parte de la terapia de la crisis de asma requiere de más estudios ${ }^{91,92}$.

\section{Ketamina}

Esta droga tiene propiedades broncodilatadoras debido a que produce un aumento de las 
catecolaminas, al inhibir su recaptación a nivel de las neuronas presinápticas. Podría tener un rol en aquellos casos de crisis de asma severa con necesidad de sedación e intubación. Se ha utilizado con éxito en pacientes con status asmático refractario a terapia estándar. La dosis es de $2 \mathrm{mg} / \mathrm{kg}$ intravenosa seguido de una infusión continua de $2 \mathrm{mg} / \mathrm{kg} / \mathrm{h}$. Un estudio que incluyó a 68 pacientes, no demostró beneficio adicional por sobre el tratamiento convencional ${ }^{92}$.

Con respecto a otros medicamentos como antileucotrienos, salbutamol endovenoso, mucolíticos o macrólidos no hay evidencias que apoyen su uso en la crisis de asma ${ }^{5,92,93}$.

\section{Educación en asma bronquial}

Como se mencionó inicialmente la educación es un pilar fundamental del tratamiento del asma bronquial junto con el tratamiento farmacológico. Para alcanzar el control de la enfermedad, eliminar las crisis y lograr una buena calidad de vida es fundamental que el paciente utilice en forma permanente su tratamiento de mantención, es decir que tenga una buena adherencia a éste. Para lograr este objetivo es prioritario que el equipo de salud realice actividades educativas formales que incluyan primero la entrega de información acerca de lo que significa tener asma y del concepto de cronicidad, de manera que los pacientes entiendan la racionalidad del uso permanente del tratamiento de mantención ${ }^{94}$.

También es muy importante explicar a los padres que el objetivo del tratamiento es aliviar los síntomas y evitar las crisis, pero en ningún caso curar la enfermedad. Otro punto crucial es educar acerca de las diferencias entre los inhaladores (tratamiento permanente versus de rescate), la correcta técnica inhalatoria, los factores gatillantes o desencadenantes de crisis y especialmente en pacientes adolescentes las técnicas de automonitoreo, puesto que muchas veces subvaloran sus síntomas ${ }^{94}$. Los pacientes asmáticos deben ser citados en forma regular a control médico y no solo cuando están con crisis, pues esta estrategia por sí sola permite mejorar el control de la enfermedad. Finalmente, todo paciente debe contar con un plan de acción escrito, personalizado, de manera que sepa como actuar frente al inicio de una crisis $^{94}$.

\section{1) Adherencia al tratamiento}

Desgraciadamente, la adherencia al tratamiento de mantención en el asma bronquial es pobre, lo cual constituye la principal causa de falta de control de la enfermedad. Se considera una buena adherencia al tratamiento de mantención cuando el paciente utiliza $80 \%$ o más de la medicación indicada. Aproximadamente $50 \%$ de niños no cumplen con su tratamiento como es debido ${ }^{95,96}$. Esta falta de adherencia al tratamiento puede ser de tipo intencional o no intencional ${ }^{97}$. Esta última puede deberse a simple olvido o a una deficiente técnica inhalatoria, en cambio en la intencional el paciente o sus cuidadores deciden conscientemente no usar la medicación, la mayoría de las veces por miedo a los efectos secundarios de los medicamentos y también por falsas creencias como una posible adicción a los inhaladores, la percepción de que el tratamiento controlador no es necesario o el rechazo al diagnóstico de asma bronquial $^{97}$. La falta de adherencia no intencional es más fácil de solucionar ya sea con recordatorios o alarmas o enseñando una buena técnica inhalatoria. La falta de adherencia intencional es más difícil de remediar. Para esto se requiere no solo de aportar al paciente información adecuada sino también de un manejo que permita cambiar conductas $^{98,99}$. En la actualidad se considera que el estándar de oro para evaluar la adherencia al tratamiento es el uso de un monitor electrónico que se adosa al inhalador y que permite saber la fecha y la hora en que el paciente usó el medicamento $^{100}$. Desgraciadamente estos dispositivos no están disponibles en nuestro país, por lo que en la práctica clínica diaria lo que se utiliza es el reporte espontaneo de los padres, que es el método menos preciso ya que la mayoría de las veces se sobrevalora el real uso de la medicación. Al momento de intentar evaluar la adherencia es muy importante ser empático y no critico y utilizar un lenguaje amigable y entendible para los padres. Formas un poco más precisas de objetivar la adherencia al tratamiento de mantención son: verificar la fecha de la última receta entregada, revisar el contador de dosis del inhalador controlador y/o medir la fracción de óxido nítrico en el aire espirado $(\mathrm{FeNO})^{101-103}$.

\section{Intervenciones especificas para mejorar adhe- rencia a tratamiento}

Las intervenciones para mejorar la adherencia al tratamiento están dirigidas al paciente y su familia, a la comunidad y a los profesionales del equipo de salud

\section{Para el paciente y familia ${ }^{\mathbf{1 0 4}}$}

- Programas de educación sobre la enfermedad y objetivos del tratamiento.

- Reforzar en cada control objetivos de tratamiento. 
- Entrenamiento personalizado en la forma de administrar los inhaladores.

- Utilizar contactos telefónicos, visita domiciliaria $\mathrm{y} / \mathrm{u}$ otro tipo de redes.

- Intervención con incentivos.

- Manejo específicos de problema de salud mental de cuidadores y pacientes.

- Adecuar horarios de tratamiento a rutina familiar y del paciente.

\section{En la comunidad ${ }^{104}$}

- Programas de educación de asma bronquial en escuelas.

\section{Del equipo de salud ${ }^{104-106}$}

- Estabilidad del equipo de salud en programas de asma bronquial.

- Educación continua al equipo de salud.

- Compartir la elección del tratamiento controlador explicando al paciente y/o cuidador los objetivos del tratamiento.

- Mejorar la comunicación con el paciente y su familia fomentando la confianza en el equipo.

- Mejorar comunicación entre la familia y equipos de salud (visitas domiciliarias, contactos telefónicos, redes).

- Recordar uso del tratamiento controlador en forma activa en cada contacto.

- Visita domiciliaria por enfermera del equipo de salud en pacientes con asma grave.

- Mensajes digitales que se gatillen en forma automática y que recuerden el uso del tratamiento de mantención.

\section{2) Técnica inhalada}

Para que el tratamiento sea eficaz no solo es importante una buena adherencia a la terapia, sino que también es fundamental que el paciente realice una técnica inhalatoria correcta que asegure la máxima llegada del medicamento a nivel bronquial. Sin embargo, vemos que la mayoría de los pacientes no son capaces de utilizar su inhalador correctamente: de acuerdo a la literatura internacional los errores en la técnica inhalada varían entre un $50 \%$ a $100 \%{ }^{107,108}$ y en un estudio prospectivo, multicéntrico realizado en nuestro país en 396 niños hospitalizados por crisis de asma se observó que $96 \%$ de ellos utilizaba la aerocámara incorrecta y que $92,8 \%$ cometía uno o más errores en la técnica inhalatoria de acuerdo a una pauta de cotejo pre establecida ${ }^{4}$. Esta realidad hace imperioso realizar actividades educativas que expliquen su uso correcto y que el equipo de salud esté capacitado, de manera de que todos, en los distintos niveles de atención, entreguen contenidos similares a los pacientes.
Nuestro propósito es que las pautas propuestas para el uso correcto de los inhaladores por este consenso SOCHINEP-SER sean difundidas y utilizadas a nivel nacional.

\section{Técnica de inhalación}

a) Inhalador presurizado

1. Niño(a) sentado(a) o de pie, con leve extensión del cuello.

2. Agitar el inhalador 5 veces.

3. Retirar la tapa del inhalador.

4. Poner el inhalador en la aerocámara, siempre en posición vertical.

5. Pedir al niño(a) que exhale lo más posible fuera de la aerocámara.

6. Poner la boquilla en la boca del niño(a), por detrás de los dientes, asegurándose que los labios queden bien sellados alrededor de la boquilla.

7. Pulsar una vez el inhalador.

8a. Pedir al niño(a)que haga una inhalación lenta y profunda por la boca hasta llenar completamente los pulmones, que contenga la respiración por 10 segundos y luego que exhale. Si el niño(a) no lo puede hacer correctamente seguir en el punto $8 \mathrm{~b}$.

8 b. Pedir al niño(a) que haga 5 respiraciones lentas y profundas.

9. Retirar la aerocámara.

10. En caso de requerir 2 inhalaciones esperar 30 segundos a 1 minuto entre una y otra y repetir el procedimiento.

11. Retirar el inhalador de la aerocámara y poner la tapa.

12. En caso de utilizar inhaladores que contengan corticoides, se recomienda enjuagar la boca.

b) Inhalador de polvo seco turbuhaler

1. Retirar la tapa.

2. Mantener el inhalador en posición vertical, con la base de color rojo abajo

3. Girar la base en un sentido hasta que tope y luego en sentido contrario.

En uno de los dos giros deberá escuchar un click.

4. Exhalar fuera del dispositivo y poner la boquilla entre los dientes y labios de manera que quede bien sellado.

5. Inhalar lento y profundo.

6. Contener la respiración por 10 segundos.

7. En caso de requerir 2 inhalaciones esperar 30 segundos a 1 minuto entre una y otra.

8. Luego de utilizar el inhalador enjuagar la boca. 
c) Inhalador de polvo seco accuhaler

1. Deslizar la tapa de manera que quede descubierta la boquilla.

2. Cargar el inhalador deslizando la palanca lateral hasta escuchar un click.

3. Exhalar fuera del dispositivo y poner la boquilla entre los dientes y labios de manera que quede bien sellado.

4. Inhalar lento y profundo.

5. Mantener la respiración por 10 segundos.

6. En caso de requerir 2 inhalaciones esperar 30 segundos a 1 min entre una y otra.

7. Luego de utilizar el inhalador enjuagar la boca.

d) Inhalador de polvo seco ellipta

1. Deslizar la tapa hasta escuchar un click, con esto queda descubierta la boquilla y al mismo tiempo el inhalador queda cargado.

2. Exhalar fuera del dispositivo y poner la boquilla entre los dientes y labios de manera que quede bien sellado.

3. Inhalar lento y profundo.

4. Contener la respiración por 10 segundos.

5. Luego de utilizar el inhalador enjuagar la boca.

\section{e) Inhalador respimat}

1. Girar la base del inhalador hasta escuchar un click con lo que el inhalador queda cargado.

2. Abrir la tapa del inhalador.

3. Exhalar fuera del dispositivo y poner la boquilla entre los dientes y labios de manera que quede bien sellado.

3. Apretar el botón dosificador y al mismo tiempo inhalar lento y profundo.

4. Contener la respiración por 10 segundos.

Cuando el dispositivo está nuevo, deben descartarse las primeras 3 dosis (girando la base del inhalador y apretando el botón dosificador, repitiendo la maniobra 3 veces).

\section{Aerocámara}

Los inhaladores presurizados son los más utilizados por los pacientes con asma a nivel mundial. Sin embargo, su administración puede ser problemática ya que la mayor parte de las partículas, emitidas a una gran velocidad, quedan impactadas en la orofaringe, lo que disminuye drásticamente su llegada a los bronquios, limitando su efecto terapéutico. En el caso de los corticoides inhalados la impactación orofaríngea favorece la aparición de efectos adversos secundarios, al ser deglutidos ${ }^{109}$. Idealmente debe existir una adecuada sincronización entre el disparo de la medicación y la inhalación, lo que habitualmente no ocurre en los pacientes pediátricos ${ }^{109}$. Por ello se hace obligatorio el uso del inhalador presurizado con aerocámara, la cual debe tener las siguientes características: poseer una boquilla y no máscara facial en la edad escolar; un volumen entre 100 a $700 \mathrm{ml}$; proveer una distancia $\geq$ de $10 \mathrm{~cm}$ entre el canister y la boca del paciente y ser valvulada, lo que permite retener el aerosol dentro de la aerocámara hasta que el paciente inhale y evitar que el aire exhalado entre a la cámara y sea reinhalado ${ }^{110}$. La aerocámara además debe ser antiestática ya que de lo contrario las cargas eléctricas que se generan en su pared atraen a las partículas del aerosol, lo que puede reducir en forma significativa la dosis disponible del medicamento inhalado ${ }^{110}$.

\section{3) Factores ambientales}

Los factores ambientales tienen un papel reconocido como gatillantes de crisis de asma. Pueden ser alérgenos a los cuales el paciente esté sensibilizado (ácaros, pólenes, caspas de animales, hongos) o contaminantes ambientales como las estufas a parafina o leña y el humo de cigarrillo. Es muy importante que los pacientes y sus cuidadores tengan conciencia de estos gatillantes de manera de evitarlos, para lo cual se debe realizar una historia ambiental exhaustiva y preguntar por exposiciones en el hogar, incluyendo todas las residencias donde el niño duerme o pasa tiempo importante ${ }^{111}$.

\section{Ácaros del polvo}

No se pueden eliminar completamente. Se puede sugerir el uso de fundas impermeables para las almohadas y colchón de la cama del niño, lavar las sábanas con agua muy caliente. Retirar los peluches de la pieza o lavarlos semanalmente, aspirar frecuentemente en lo posible con aspiradora con filtro HEPA, (High Efficiency Particle Arresting) utilizar trapeador húmedo, evitar el uso de humidificadores, retirar alfombras y muebles tapizados del dormitorio del paciente ${ }^{111}$.

\section{Caspas de animales}

Recomendar mantener a las mascotas fuera de la casa y si esto no es posible al menos mantenerlas fuera del dormitorio. Bañarlas al menos 2 veces por semana ${ }^{111}$.

\section{Hongos}

Se debe poner énfasis en el control de las fuentes de humedad de la casa: verificar filtraciones, controlar la humedad interior, usar deshumidifi- 
cador para mantener la humedad relativa en el interior bajo $50 \%$, ventilar baños y evitar empapelado de paredes en áreas húmedas y el uso de humidificadores ${ }^{111}$.

\section{Humo de tabaco}

El humo del tabaco y del cigarrillo electrónico contienen muchos químicos e irritantes tóxicos. Los niños asmáticos expuestos tienen mayor frecuencia de crisis de asma e infecciones respiratorias de la vía aérea inferior. No basta fumar fuera de la casa evitando el contacto directo con el niño. Esto no es suficiente ya que se ha demostrado que el contacto con humo de tercera mano (humo que se adhiere a la ropa, cabello, tapiz del automóvil y muebles) también es nocivo ${ }^{111}$.

\section{Contaminación del aire}

El ozono y material particulado pueden aumentar los síntomas de asma y gatillar crisis. Se debe estar atentos a los niveles de calidad del aire y reducir o evitar la actividad física cuando el índice de calidad del aire esté en rango peligroso ${ }^{111}$.

\section{Pólenes}

Evitar el polen por completo puede ser difícil durante la época de primavera, pero se pueden tomar algunas medidas para reducir la exposición, como evitar salir al aire libre en días con altos niveles polínicos, especialmente si hay mucho viento, ducharse luego de realizar actividades al aire libre, conducir con ventanas del automóvil cerradas y usar mascarilla en situaciones especiales como cuando es inevitable el contacto con altos niveles de este alérgeno ${ }^{112}$.

\section{4) Automonitoreo}

Las técnicas de automonitoreo son especialmente útiles en los pacientes que subvaloran sus síntomas, como sucede en los adolescentes. Las herramientas de automonitoreo más utilizadas son los cuestionarios y la flujometría. Dentro de los cuestionarios, los más utilizados son los que miden el control de la enfermedad como el Asthma Control Test (ACT) y el Asthma Control Questionnaire (ACQ) ${ }^{113,114}$. Existen otros cuestionarios como el Composite Asthma Severity Index (CASI) que tiene la capacidad de determinar además el nivel de severidad del asma. Este es un cuestionario considerado multidimensional ya que no solo incluye la presencia de síntomas, sino que también incluye la función pulmonar y la presencia de crisis ${ }^{115}$. Por otro lado, la flujometría se puede utilizar para evaluar la respuesta al tratamiento, el nivel de control de la enfermedad y el riesgo de crisis. La guía GINA en la actualidad recomienda su uso solo en pacientes con asma grave y para aquellos con escasa percepción de síntomas 5 .

\section{5) Control médico periódico}

El control médico periódico es fundamental para el éxito del tratamiento de asma. La frecuencia de las visitas dependerá del control de la enfermedad ${ }^{5}$. En cada visita se debe:

a) Preguntar al paciente si tiene alguna duda o preocupación: en esta instancia se debe resolver todas las inquietudes y complementar con actividades educativas. En esta tarea participa todo el equipo de salud.

b) Evaluar el control de la enfermedad: se debe valorar el nivel de control de los síntomas, la presencia de factores de riesgo, ocurrencia de crisis y si la respuesta al tratamiento fue adecuada. Indagar acerca de la frecuencia del uso de medicamentos de rescate, visitas a servicio de urgencia y/o hospitalización, uso de corticoides sistémicos e inasistencia escolar. Aplicar cuestionarios de control del asma y evaluar flujometría si el paciente la utiliza. Finalmente se debe chequear la presencia de comorbilidades.

c) Evaluar problemas con el tratamiento: observar la técnica inhalada y corregir si es necesario, evaluar la adherencia al tratamiento de mantención y revisar el plan de acción escrito con el paciente.

d) Dejar agendado el próximo control médico: cada cierto tiempo es deseable evaluar la calidad de vida del paciente y sus cuidadores, mediante cuestionarios e indicar en forma anual la vacuna antigripal.

\section{6) Plan de acción (ver Anexo)}

Corresponde a un plan de acción escrito, personalizado para cada paciente y que entrega educación y guía para el automanejo en caso de empeoramiento de los síntomas de asma. Habitualmente se configura con los colores del semáforo.

Zona verde: describe un control adecuado y la indicación en este caso corresponde al uso del tratamiento de mantención.

Zona amarilla: describe la pérdida de control, por lo que se debe indicar la conducta a seguir y qué medicamento de rescate utilizar.

Zona roja: indica la presencia de síntomas graves que requieren de atención médica inmediata.

El uso del plan de acción escrito se ha asociado a una reducción de $70 \%$ en la mortalidad por asma y al aplicarlo en conjunto con activi- 
dades educativas y control médico periódico, reduce en forma significativa la utilización de recursos en salud, el ausentismo escolar y los síntomas de asma, mejorando además la calidad de vida ${ }^{116}$. Debido a esto es que todas las guías internacionales recomiendan que la totalidad de los pacientes con asma reciba un plan de acción, pese a lo cual su indicación en la realidad es de solo un $30 \%{ }^{116}$. Dado el alto nivel de evidencia que apoya el uso del plan de acción escrito es que esta Comisión recomienda su uso en todo niño con asma.

\section{Conclusiones}

Se debe sospechar el diagnóstico de asma bronquial en todo escolar con episodios recurrentes de obstrucción bronquial. Esta sospecha debe confirmarse siempre con estudios de función pulmonar e iniciar tan pronto sea posible un tratamiento que permita alcanzar el control de la enfermedad. El tratamiento de elección es el uso de corticoides inhalados permanentes en conjunto con un programa de educación y seguimiento estrecho.

Anexo. Plan de acción en asma bronquial

Nombre:

Fecha:

\section{Zona Verde}

Me siento bien, no tengo tos ni silbido al pecho, ni sensación de pecho apretado ni dificultad para respirar ni de día ni de noche.

Puedo realizar mis actividades habituales sin problemas

$\rightarrow$ Solo debo usar en forma permanente mi tratamiento habitual: Medicamento y dosis:

\section{Zona Amarilla}

Mi asma está empeorando, tengo tos, silbido al pecho, sensación de pecho apretado, dolor al pecho y/o dificultad para respirar

a) Si mi tratamiento habitual es el uso permanente de corticoides inhalados (Budesonida o Fluticasona) o la mezcla de Fluticasona/Salmeterol o Fluticasona/Vilanterol

Debo usar:

Salbutamol 2 puff con aerocámara

Si los síntomas pasan, mantener salbutamol 2 puff cada $6 \mathrm{~h}$ y pedir hora con mi doctor dentro de los próximos días

Si los síntomas no pasan, usar salbutamol 6 puff cada 20 minutos por 3 veces

Si los síntomas pasan, debo seguir usando salbutamol 2 puff cada $4 \mathrm{~h}$ y pedir hora con mi médico tratante lo más pronto posible
Si los síntomas no pasan o luego de haber cedido reaparecen, debo acudir lo más pronto posible al servicio de urgencia 
Anexo. Plan de acción en asma bronquial (continuación)

$\rightarrow$ b) Si mi tratamiento habitual es el uso de la mezcla Budesonida/Formoterol

Debo usar:

La mezcla de Budesonida/Formoterol 1 a 8 inhalaciones extras

Si los síntomas pasan, mantener el uso de la mezcla de Budesonida/Formoterol 1 a 8 inhalaciones al día - Beclometasona/Salbutamol 2 puff cada $4 \mathrm{~h}$ y pedir hora con mi doctor para los próximos días
Si los síntomas no pasan o luego de haber cedido reaparecen debo acudir al servicio de urgencia lo más pronto posible

c) Si estoy sin tratamiento de mantención debo usar la mezcla de Budesonida/Formoterol 1 a 8 inhalaciones o la mezcla de Beclometasona/Salbutamol 6 puff cada 20 min por $1 \mathrm{~h}$

Si los síntomas pasan, mantener el uso de la mezcla de Budesonida/Formoterol 1 a 8 inhalaciones al día - Beclometasona/ Salbutamol 2 puff cada 4 h y pedir hora con mi doctor para los próximos días
Si los síntomas no pasan o luego de haber cedido reaparecen debo acudir al servicio de urgencia lo más pronto posible

\section{Zona Roja}

Tengo una crisis de asma severa, no puedo hablar porque me falta el aire, debo hacer mucho esfuerzo para respirar, mi respiración es rápida y/o tengo coloración azul en los labios

\section{$\rightarrow$ iiiiESTO ES UNA URGENCIA MÉDICA!!!!}

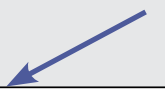

Si mi tratamiento habitual es el uso permanente de Budesonida, Fluticasona o la mezcla Fluticasona/Salmeterol o Fluticasona/Vilanterol debo usar Salbutamol 6 puff cada 20 min hasta llegar lo más rápidamente posible al servicio de urgencia

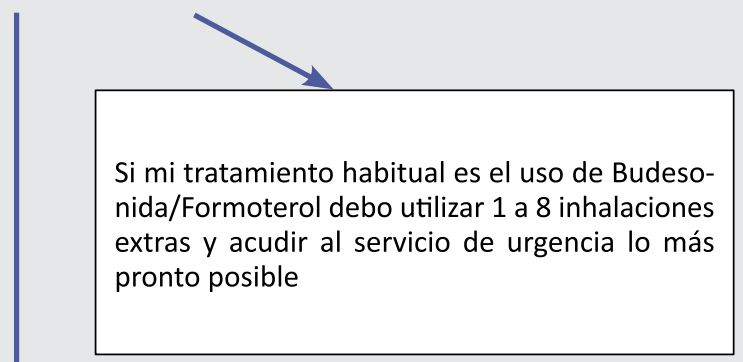

Si estoy sin tratamiento de mantención debo usar la mezcla de Budesonida/Formoterol 1 a 8 inhalaciones o la mezcla de Beclometasona/ Salbutamol 6 puff cada 20 min hasta llegar lo más rápidamente posible al servicio de urgencia 


\section{Bibliografía}

1.- ASMA - WORLD HEALTH ORGANIZATION https://www.who.int/es/news-room/fact-sheets/detail/ asthma (accesado en marzo de 2020).

2.- MALLOL J, AGUIRRE V, AGUILAR P, CALVO M, AMARALES L, ARELLANO P, et al. Cambios en la prevalencia del asma en escolares chilenos entre 1994 y 2002. Rev Med Chile 2007; 135: 580-6.

3.- $\quad$ HERRERA AM, CAVADA G, MAÑALICH J. Hospitalizaciones por asma infantil en Chile: 2001-2014. Rev Chil Ped 2017; 88: 602-7.

4.- HERRERA AM, BRAND P, CAVADA G, KOPPMANN A, RIVAS M, MACKENNEY J. Hospitalizations for asthma exacerbation in Chilean children: A multicenter observational study. Allergol Immunopathol (Madr). 2018; 46: 533-8.

5.- GLOBAL INITIATIVE FOR ASTHMA. Global Strategy for Asthma Management and Prevention 2019. https:/ginasthma.org/wp-content/uploads/2019/06/ GINA-2019-main-report-June-2019-wms.pdf (accesado en marzo de 2020).

6.- GEMA Guía española para el manejo del asma. https:// www.semg.es/images/documentos/GEMA 4 4.pdf

7.- BUSH A, FLEMING L. Is asthma overdiagnosed? Arch Dis Child 2016; 101: 688-9.

8.- QUANJER PH, STANOJEVIC S, COLE TJ, BAUR $\mathrm{X}$, HALL GL, CULVER BH, et al. Multiethnic reference values for spirometry for the 3-95 yr age range: the Global Lung Function 2012 equations. Eur Respir J 2012; 40: 1324-43.

9.- GUTIÉRREZ CM, BEROIZA T, BORZONE G, CAVIEDES I, CÉSPEDES J, CORRALES R, et al. Espirometría: manual de procedimientos SER Chile. Rev Chil Enf Respir 2018; 34: 171-88.

10.- GRAHAM BL, STEENBRUGGEN I, MILLER MR, BARJAKTAREVIC IZ, COOPER BG, HALL GL, et al. Standardization of Spirometry 2019 Update. Ann Official American Thoracic Society and European Respiratory Society Technical Statement. Am J Respir Crit Care Med 2019; 200: e70-e88.

11.- CAUSSADE S, SAAVEDRA M, BARRIENTOS H, LINARES M, AGUIRRE V, PUPPO H, et al. Actualización en espirometría y curva flujo/volumen en escolares y adolescentes. Neumol Pediatr 2019; 14: 41-51.

12.- MOELLER A, CARLSEN KH, SLY PD, BARALDI E, PIACENTINI G, PAVORD I, et al. Monitoring asthma in childhood: lung function, bronchial responsiveness and inflammation. Eur Respir Rev 2015; 24: 204-15.

13.- MCGEACHIE MJ, YATES KP, ZHOU X, GUO F, STERNBERG AL, VAN NATTA ML, et al. Patterns of growth and decline in lung function in persistent childhood asthma. N Engl J Med 2016; 374: 1842-52.

14.- COATES AL, WANGER J, COCKCROFT DW, CUL-
VER BH, CARLSEN KH, DIAMANT Z, et al. ERS technical standard on bronchial challenge tests: general considerations and performance of methacholine challenge tests. Eur Respir J 2017; 49: 1601526.

15.- UBILLA C, SAAVEDRA M, LEWINSON D, LINARES M, ÁLVAREZ C, BARRIENTOS H, et al. Update in Methacholine Challenge Test in Children. Neumol Pediatr 2019; 14: 175-9.

16.- NATIONAL INSTITUTE FOR HEALTH AND CARE EXCELLENCE (NICE) GUIDELINE 2017. Asthma: Diagnosis, Monitoring and Chronic Asthma Management. https://www.nice.org.uk/guidance/ng80/resources/asthma-diagnosis-monitoring-and-chronicasthmamanagement-pdf-1837687975621 (accesado en marzo de 2020).

17.- BRITISH GUIDELINE ON THE MANAGEMENT OF ASTHMA 2019. file://Users/amherrerag/Downloads/BTS SIGN\%20Guideline $\% 20$ for $\% 20$ the $\% 20$ management $\% 20$ of\%20asthma\%202019.pdf (accesado en marzo de 2020).

18.- HALLSTRAND TS, LEUPPI JD, JOOS G, HALL GL, CARLSEN KH, KAMINSKY DA, et al. ERS technical standard on bronchial challenge testing. pathophysiology and methodology of indirect airway challenge testing. Eur Respir J 2018; 52: 1801033.

19.- PARSONS JP, HALLSTRAND TS, MASTRONARDE JG, KAMINSKY DA, RUNDELL KW, HULL JH, et al. Ann Official American Thoracic Society Clinical Practice Guideline: Exercise-induced Bronchoconstriction. Am J Respir Crit Care Med 2013; 187: 1016-27.

20.- GALLUCI M, CARBONARA P, PACILLI AMG, DI PALMO E, RICCI G, NAVA S. Use of Symptoms Scores, Spirometry and Other Pulmonary Function Testing for Asthma Monitoring. Front Pediatr 2019; 7: 54.

21.- DWEIK RA, BOGGS PB, ERZURUM SC, IRVIN CG, LEIGH MW, LUNDBERG JO, et al. An official ATS clinical practice guideline: interpretation of exhaled nitric oxide levels (FENO) for clinical applications. Am J Respir Crit Care Med 2011; 184: 602-15.

22.- MALINOVSCHI A, FONSECA JA, JACINTO T, ALVING K, JANSON C. Exhaled nitric oxide levels and blood eosinophil counts independently associated with wheeze and asthma events in National Health and Nutrition Examination Survey Subjects. J Allergy Clin Immunol 2013; 132: 821-7.e1-5.

23.- LO DKH, BEARDSMORE CS, ROLAND D, RICHARDSON M, YANG Y, DANVERS L, et al. Lung function and asthma control in school-age children managed in UK primary care: a cohort study. Thorax 2020; 75: 101-7.

24.- PIJNENBURG MW, BAKKER EM, HOP WC, DE JONGSTE JC. Titrating steroids on exhaled nitric oxide in children with asthma. A Randomized Controlled Trial. Am Respir Crit Care Med 2005; 172: 831-6.

25.- PIJNENBURG MW, HOFHUIS W, HOP WC, DE JONGSTE JC. Exhaled nitric oxide predicts asthma 
relapse in children with clinical asthma remission. Thorax 2005; 60: 215-8.

26.- ZACHARASIEWICS A, WILSON N, LEX C, ERIN EM, LI AM, HANSEL T, et al. Clinical use of noninvasive measurements of airway inflammation in steroid reduction in children. Am J Respir Crit Care Med 2005; 171: 1077-82.

27.- ANDERSON HM, LEMANSKE RFJr, ARRON JR, HOLWEG CTJ, RAJAMANICKAM V, GANGNON $\mathrm{R}$, et al. Relationships among aeroallergen sensitization, peripheral blood eosinophils, and periostin in pediatric asthma development. J Allergy Clin Immunol 2017; 139: 790-6.

28.- BOSSLEY CJ, FLEMING L, GUPTA A, REGAMEY N, FRITH J, OATES T, et al. Pediatric severe asthma is characterized by eosinophilia and remodeling without Th2 cytokines. J Allergy Clin Immunol 2012; 129: $974-82$.

29.- TRAN TN, KHATRY DB, KE X, WARD CK, GOSSAGE D. High blood eosinophil count is associated with more frequent asthma attacks in asthma patients. Ann Allergy Asthma Immunolol 2014; 113: 19-24.

30.- ULLMANN N, BOSSLEY CJ, FLEMING L, SILVESTRI M, BUSH A, SAGLANI S. Blood eosinophil counts rarely reflect airway eosinophilia in children with severe asthma. Allergy 2013; 68: 402-6.

31.- SÁNCHEZ-GARCÍA S, HABERNAU MENA A, QUIRCE S. Biomarkers in inflammometry pediatric asthma: utility in daily clinical practice. Eur Clin Respir J 2017; 4: 1356160. doi: 10.1080/20018525.2017.1356160.

32.- ARRON JR, IZUHARA K. Asthma biomarkers: what constitutes a "gold standard"? Thorax 2015; 70: 105-7.

33.- BANTZ SK, ZHU Z, ZHENG T. The role of Vitamin D in Pediatric Asthma. Ann Pediatr Child Health 2015; 3: 1032.

34.- CASSIM R, RUSSELL MA, LODGE CJ, LOWE AJ, KOPLIN JJ, DHARMAGE SC. The role of circulating 25 Hidroxyvitamin D in asthma: a systematic review. Allergy 2015; 70: 339-54.

35.- ZHANG Y, LEUNG DYM, GOLAVA E. Anti-inflammatory and corticosteroid enhancing actions of vitamin D in monocytes of patients with steroid-resistant and those with steroid sensitive asthma. J Allergy Clin Inmunol 2014; 133: 1744-52.

36.- JOLLIFFE DA, GREENBERG L, HOOPER RL, GRIFFITHS CJ, CAMARGO CA JR, KERLEY CP, et al. Vitamin D supplementation to prevent asthma exacerbations: a systematic review and meta-analysis of individual participant data. Lancet Respir Med 2017; 5: 881-90.

37.- BOUSQUET J, MANTZOURANIS E, CRUZ AA, AÏT-KHALED N, BAENA-CAGNANI CE, BLEECKER ER, at al. Uniform Definition of Asthma Severity, Control, and Exacerbations: Document Presented for the World Health Organization Consultation on
Severe Asthma. Allergy Clin Immunol 2010; 126: 926-38.

38.- BRACKEN M. FLEMING L, HALL P, VAN STIPHOUT N, BOSSLEY C, BIGGART E, et al. The importance of nurse-led home visits in the assessment of children with problematic asthma. Arch Dis Child 2009; 94: 780-4.

39.- PAPI A, BRIGHTLING C, PEDERSEN S, REDDEL H. Asthma. Lancet 2018; 391: 783-800.

40.- SZEFLER S, CHIPPS B. Challenges in the treatments of asthma in children and adolescents. Ann Allergy Asthma Immunol 2018; 120: 382-8.

41.- HERRERA AM, ABARA S, ÁLVAREZ C, ASTUDILLO C, CORRALES R, CHALA E, et al. Consenso chileno SER-SOCHINEP para el manejo del asma severo en el niño. Rev Chil Enferm Respir 2016; 32 : 100-12.

42.- LEMANSKE RF, MAUGER DT, SORKNESS CA, PHARM D, JACKSON DJ, BOEHMER SJ, et al. Step-up Therapy for Children with Uncontrolled Asthma While Receiving Inhaled Corticosteroids. N Engl J Med 2010; 362: 975-85.

43.- RODRIGO G, NEFFEN H. Efficacy and safety of tiotropium in school-age children with moderate to severe symtomatic asthma: A sistematic review. Pediatr Allergy Immunol 2017; 28: 573-8.

44.- SUISSA S, ERNST P, BOIVIN JF, HORWITZ RI, HABBICK B, COCKROFT D, et al. A Cohort Analysis of Excess Mortality in Asthma and the Use of Inhaled- B Agonists. Am J Respir Crit Care Med. 1994; 149: 604-10.

45.- ABRAMSON MJ, BAILEY MJ, COUPER FJ, DRIVER JS, DRUMMER OH, FORBES AB, et al. Are asthma medications and management related to deaths from asthma? Am J Respir Crit Care Med. 2001; 163 : 12-8.

46.- $\quad$ STANFORD RH, SHAH MB, D'SOUZA AO, DHAMANE AD, SCHATZ M. Short-acting $\beta$-agonist use and its ability to predict future asthma-related outcomes. Ann Allergy Asthma Immunol. 2012; 109: 403-7.

47.- SUISSA S, ERNST P, KEZOUH A. Regular use of inhaled corticosteroids and the long term prevention of hospitalisation for asthma. Thorax. 2002; 57: 880-4.

48.- SUISSA S, ERNST P, BENAYOUN S, BALTZAN M, CAI B. Low-dose inhaled corticosteroids and the prevention of death from asthma. N Engl J Med. 2000; 343: 332-6.

49.- $\quad$ SOBIERAJ DM, WEEDA ER, NGUYEN E, COLEMAN CI, WHITE CM, LAZARUS SC, et al. Association of Inhaled Corticosteroids and Long-Acting $\beta$-Agonists as Controller and Quick Relief Therapy With Exacerbations and Symptom Control in Persistent Asthma: A Systematic Review and Meta-analysis. JAMA. 2018; 319: 1485-96.

50.- O'BYRNE PM, FITZGERALD M, BATEMAN E, BARNES PJ, ZHONG N, KEEN C, et al. Inhaled 
Combined Budesonide-Formoterol as Needed in Mild Asthma. N Engl J Med. 2018; 378: 1865-76.

51.- BATEMAN ED, REDDEL HK, O’BYRNE PM, BARNES PJ, ZHONG N, KEEN C, et al. As-Needed Budesonide-Formoterol versus Maintenance Budesonide in Mild Asthma. N Engl J Med. 2018; 378: 1877- 87.

52.- MCGHEE S. Biologics in pediatric lung disease. Curr Opin Pediatr 2018; 30: 366-71.

53.- HERRERA AM, HERNÁNDEZ J, PERILLÁN JA, LEZANA V, ÁLVAREZ C, MARINOVIC MA, et al. Tratamiento del asma severo con Omalizumab: experiencia pediátrica en Chile y revisión de la literature. Rev Chil Enferm Respir 2016; 32: 160-8.

54.- JOLLIFFE D, GRIFFITHS C, CAMARGO C, KERLEY C, JENSEN M, MAUGER D, et al. Vitamin D supplementation to prevent asthma exacerbations: a systematic review and meta-analysis of individual participant data. Lancet Respir Med 2017; 5: 881-90.

55.- DINAKAR C, CHIPPS B. Clinical tools to assess asthma control in children. Pediatrics 2017; 139 e20163438. doi: https://doi.org/10.1542/peds.20163438 .

56.- MAITRAA. Severe asthma: challenges and pitfalls in management. Indian J Pediatr 2018; 85: 763-72.

57.- BUSH A. Asthma: What's new, and what should be old but is not! Pediatr Respirol Crit Care Med 2017; 1: 2-10.

58.- FITZGERALD JM. Targeting lung attacks. Thorax 2011; 66: 365-6.

59.- SAGLANI S, FLEMING L, SONNAPPA S, BUSH A. Advances in the aetiology, management, and prevention of acute asthma attacks in children. Lancet Child Adolesc Health 2019; 3: 354-64.

60.- DONDI A, CALAMELLI E, PICCINNO V, RICCI G, CORSINI I, BIAGI C, et al. Acute Asthma in the Pediatric Emergency Department: Infections Are the Main Triggers of Exacerbations. BioMed Res Int 2017; 2017: 9687061. doi: 10.1155/2017/9687061.

61.- MERCKX J, DUCHARME FM, MARTINEAU C, ZENNEK R, GRAVEL J, CHALUT D, et al. Respiratory viruses and treatment failure in children with asthma exacerbation. Pediatrics 2018; 142: e20174105. doi: 10.1542/peds.2017-4105.

62.- BIZZINTINO J, LEE WM, LAING IA, VANG F, PAPPAS T, ZHANG G, et al. Association between human rhinovirus $\mathrm{C}$ and severity of acute asthma in children. Eur Respir J 2011; 37: 1037-42.

63.- LOOI K, BUCKLEY AG, RIGBY PJ, GARRATT LW, IOSIFIDIS T, ZOSKY GR, et al. Effects of human rhinovirus on epithelial barrier integrity and function in children with asthma. Clin Exp Allergy 2018; 48: 513-24.

64.- BONNELYKKE K, COLEMAN AT, EVANS MD, THORSEN J, WAAGE J, VISSING NH, et al. Cadherin- related Family Member 3 Genetics and Rhinovirus
C Respiratory Illnesses. Am J Respir Crit Care Med 2018; 197: 589-94.

65.- BASNET S, BOSHKOV YA, BROCKMAN-SCHNEIDER RA, KUIPERS I. AESIF SW, JACKSON DJ, et al. CDHR3 Asthma-Risk Genotype Affects Susceptibility of Airway Epithelium to Rhinovirus C Infections. Am J Respir Cell Mol Biol. 2019; 61: 450-8.

66.- KLOEPFER KM, LEE WM, PAPPAS TE, KANG TJ, VRTIS RF, EVANS MD, et al. Detection of pathogenic bacteria during rhinovirus infection is associated with increased respiratory symptoms and asthma exacerbations. J Allergy Clin Immunol 2014; 133: 1301-7.

67.- ERBAS B, JAZAYERI M, LAMBERT KA, KATELARIS CH, PRENDERGAST LA, THAM R, et al. Outdoor pollen is a trigger of child and adolescent asthma emergency department presentations: A systematic review and meta-analysis. Allergy 2018; 73: 1632-41.

68.- HEW M LEE J, SUSANTO NH, PRASAD S, BARDIN PG, BARNES S, RUANE L, et al. The $2016 \mathrm{Mel}-$ bourne thunderstorm asthma epidemic: risk factors for severe attacks requiring hospital admission. Allergy 2019; 74: 122-30.

69.- RAMSAHAI JM, HANSBRO PM, WARK PAB. Mechanisms and Management of Asthma Exacerbations. Am J Respir Crit Care Med 2019; 199: 423-32.

70.- COHEN HA, BLAU H, HOSHEN M, BATAT E, BALICER RD. Seasonality of Asthma: A Retrospective Population Study. Pediatrics 2014; 133: e923-e932.

71.- SMITH SR, BATY JD, HODGE D. Validation of the Pulmonary Score: an asthma severity score for children. Acad Emerg Med 2002; 9: 99-104.

72.- CHANDRAKALA PAC. RAMESH, CHAITRA RAO B. Validation of Pulmonary Score in the Evaluation of Acute Exacerbation of Asthma. Acad Emerg Med 2002; 9: 99-104.

73.- GORELICK MH, STEVENS MW, SCHULTZ, SCRIBANO PV. Difficulty in obtaining peak expiratory flow measurements in children with acute asthma. Pediatr Emerg Care 2004; 20: 22-6.

74.- CAMARGO C, RACHELEFSKY G, SCHATZ M. Managing Asthma Exacerbations in the Emergency Department Summary of the National Asthma Education and Prevention Program Expert Panel Report 3 Guidelines for the Management of Asthma Exacerbations. Proc American Thoracic Society 2009; 6: 357-66.

75.- ALLIE EH, DINGLE HE, JOHNSON WN, BIRNBAUM JR, HILMES MA, SINGH SP, et al. ED chest radiography for children with asthma exacerbation is infrequently associated with change of management. Am J Emerg Med 2018; 36: 769-73.

76.- CASTRO-RODRÍGUEZ JA, RODRIGO GJ. Betaagonist through metered-dose inhaler with valved holding chamber versus nebulizer for acute exacerbation of wheezing or asthma in children under 5 years of age 
A systematic review with meta-analysis. J Ped. 2004; 145: $172-7$.

77.- CATES CJ, WELSH EJ, ROWE BH. Holding chambers (spacers) versus nebulisers for beta-agonist treatment of acute asthma. Cochrane database Syst rev. Cochrane Database Syst Rev. 2013; 9: CD000052.

78.- KHINE H, FUCHS SM, SAVILLE AL. Continuous vs intermittent nebulized albuterol for emergency management of asthma. Acad Emerg Med. 1996; 3: 1019-24.

79.- $\quad$ RODRIGO GJ, CASTRO-RODRÍGUEZ JA. Anticholinergics in the treatment of children and adults with acute asthma: a systematic review with meta-analysis. Thorax 2005; 60: 740-6.

80.- GRIFFITHS B, DUCHARME FM. Combined inhaled anticholinergics and short-acting beta2-agonists for initial treatment of acute asthma in children. Cochrane Database Syst Rev. 2013; 8: CD000060.

81.- VEZINA K, CHAUHAN BF, DUCHARME FM. Inhaled anticholinergics and short-acting beta(2)-agonists versus short- acting beta 2 -agonists alone for children with acute asthma in hospital. Cochrane Database Syst Rev 2014; 7: CD010283.

82.- BHOGAL SK. A question of time: systemic corticosteroids in managing acute asthma in children. Curr Opin Pulm Med. 2013; 19: 73-8.

83.- NORMANSELL R, KEW KM, MANSOUR G. Different oral corticosteroid regimens for acute asthma. Cochrane Database of Systematic Reviews 2016, Issue 5. Art. No.: CD011801. DOI: 10.1002/14651858. CD011801.pub2.

84.- EDMONDS ML, MILAN SJ, BRENNER BE, CAMARGO CA Jr, ROWE BH. Inhaled steroids for acute asthma following emergency department discharge. Cochrane Database Syst Rev. 2012; 12: CD002316. doi: 10.1002/14651858.CD002316.pub2.

85.- $\quad$ SU Z, LI R, GAI Z. Intravenous and nebulized magnesium sulfate for treating acute asthma in children: a systematic review and meta-analysis. Pediatr Emerg Care. 2018; 34: 390-5.

86.- TORRES S, STICCO N, BOSCH JJ, IOLSTER T, SIABA A, ROCCA RIVAROLA M, et al. Effectiveness of magnesium sulfate as initial treatment of acute severe asthma in children, conducted in a tertiary-level university hospital: a randomized, controlled trial. Arch Argent Pediatr. 2012; 110: 291-6.

87.- GRIFFITHS B, KEW KM. Intravenous magnesium sulfate for treating children with acute asthma in the emergency department. Cochrane Database Syst Rev. 2016 Apr 29; 4: CD011050. doi: 10.1002/14651858. CD011050.pub2.

88.- POWELL CV, KOLAMUNNAGE-DONA R, LOWE J, BOLAND A, PETROU S, DOULL I, et al. Magnesium Trial in Children (MAGNETIC): a randomised, placebo-controlled trial and economic evaluation of nebulised magnesium sulphate in acute severe asthma in children. Health Technol Assess. 2013; 17 (45): v-vi, 1-216.

89.- CARSON KV, USMANI ZA, SMITH BJ. Noninvasive ventilation in acute severe asthma: current evidence and future perspectives. Curr Opin Pulm Med. 2014; 20: 118-23.

90.- SOAR J, PUMPHREY R, CANT A, CLARKE S, CORBETT A, DAWSON P, et al. Working Group of the Resuscitation Council (UK). Emergency treatment of anaphylactic reactions-guidelines for healthcare providers. Resuscitation. 2008; 77: 157-69.

91.- NIEVAS IF, ANAND KJ. Severe acute asthma exacerbation in children: a stepwise approach for escalating therapy in a pediatric intensive care unit. J Pediatr Pharmacol Ther. 2013; 18: 88-104.

92.- KONINCKX M, BUYSSE C, DE HOOG M. Management of status asthmaticus in children. Paediatr Respir Rev 2013; 14: 78-85.

93.- PARDUE JONES B, FLEMING GM, OTILLIO JK, ASOKAN I, ARNOLD DH. Pediatric acute asthma exacerbations: Evaluation and management from emergency department to intensive care unit. J Asthma. 2016; 53: 607-17.

94.- BROUWER AFJ, BRAND PLP. Asthma education and monitoring: what has been shown to work. Paediatr Respir Rev. 2008; 9: 193-9.

95.- MORTON RW, EVERARD ML, ELPHICK HE. Adherence in childhood asthma: the elephant in the room. Arch Dis Child 2014; 99: 949-53.

96.- BOULET L-P, VERVLOET D, MAGAR Y, FOSTER JM. Adherence: the goal to control asthma. Clin Chest Med 2012; 33: 405-17.

97.- RAU JL. Determinants of Patient Adherence to an Aerosol Regimen. Respir Care 2005; 50: 1346-56.

98.- CLARK NM, MITCHELL HE, RAND CS. Effectiveness of Educational and Behavioral Asthma Interventions. Pediatrics 2009; 123: S185-S192.

99.- MOULLEC G, GOUR-PROVENCAL, BACON SL, CAMPBELL TS, LAVOIE KL. Efficacy of interventions to improve adherence to inhaled corticosteroids in adult asthmatics: Impact of using components of the chronic care model. Respir Med 2012; 106: 121125.

100.- JOCHMANN A, ARTUSIO L, JAMALZADEH A, NAGAKUMAR P, DELGADO-ECKERT E, SAGLANI S, et al. Electronic monitoring of adherence to inhaled corticosteroids: an essential tool in identifying severe asthma in children. Eur Respir J. 2017; 50: 1700910. doi: 10.1183/13993003.00910-2017.

101.- BOULET L-P, VERVLOET D, MAGAR Y, FOSTER JM. Adherence: the goal to control asthma. Clin Chest Med 2012; 33: 405-17.

102.- ULRIK CS, BACKER V, SOES-PETERSEN U, LANGE P, HARVING H, PLASCHKE PP. The patient's perspective: adherence or non-adherence to asthma controller therapy? J Asthma 2006; 43: 701-4. 
103.- MCNICHOLL DM, STEVENSON M, MCGARVEY LP, HEANEY LG. The utility of fractional exhaled nitric oxide suppression in the identification of nonadherence in difficult asthma. Am J Respir Crit Care Med 2012; 186: 1102-8.

104.- BOOSTER GD, OLAND AA, BENDER BG. Treatment Adherence in Young Children with Asthma. Immunol Allergy Clin North Am 2019; 39: 233-42.

105.- OTSUKI M, EAKIN MN, RAND CS, BUTZ AM, HSU VD, ZUCKERMAN IH, et al. Adherence feedback to improve asthma outcomes among inner-city children: a randomized trial. Pediatrics 2009; 124: 1513-21.

106.- WILLIAMS LK, PETERSON EL, WELLS K, CAMPBELL J, WANG M, CHOWDHRY VK, et al. A cluster-randomized trial to provide clinicians inhaled corticosteroid adherence information for their patients with asthma. J Allergy Clin Immunol 2010; 126: 225 31 .

107.- ROMÁN-RODRÍGUEZ A M, METTING B E, MARINA GARCÍA-PARDO, KOCKSC J, VAN DER MOLEN T. Wrong inhalation technique is associated to poor asthma clinical outcomes. Is there room for improvement? Curr Opin Pulm Med 2019; 25: 18-26.

108.- SAMADY W, RODRÍGUEZ VA, GUPTA R, PALAC H, KARAMANIS M, PRESS VG. Critical Errors in Inhaler Technique Among Children Hospitalized With Asthma. J Hosp Med 2019; 14: 361-5.

109.- ANDERSONA G, JOHNSON N, MULGIRIGAMAB A, AGGARWALC B. Use of spacers for patients treated with pressurized metered dose inhalers: focus on the VENTOLIN ${ }^{\text {TM }}$ Mini Spacer. Expert Opin Drug Deliv. 2018; 15: 419-30.
110.- VINCKEN W, LEVY ML, SCULLION J, USMANI OS, DEKHUIJZEN PNR, CORRIGAN CJ. Spacer devices for inhaled therapy: why use them, and how? ERJ Open Research. 2018; 4: 00065-2018.

111.- Manejo ambiental del asma pediátrico. https://www. niehs.nih.gov/health/materials/environmental_management_of_pediatric_asthma_guidelines_for_health_ care_providers_spanish_508.pdf (accesado en marzo de 2020).

112.- National Asthma Council Australia https://www. nationalasthma.org.au/living-with-asthma/resources/ patients-carers/factsheets/pollen-triggers-my-asthmaand- allergies (accesado en marzo de 2020).

113.- JIA CE, ZHANG HP, LV Y, LIANG R, JIANG YQ, POWELL H, et al. The Asthma Control Test and Asthma Control Questionnaire for assessing asthma control: Systematic review and meta-analysis. J Allergy Clin Immunol. 2013; 13: 695-703.

114.- SCHULER M, FALLER H, WITTMANN M, SCHULTZ K. Asthma Control Test and Asthma Control Questionnaire: factorial validity, reliability and correspondence in assessing status and change in asthma control. J Asthma. 2016; 53: 438-45.

115.- WILDFIRE JJ, GERGEN PJ, SORKNESS CA, MITCHELL HE, CALATRONI A, KATTAN M, et al. Development and Validation of the Composite Asthma Severity Index - An Outcome Measure for use in Children and Adolescents. J Allergy Clin Immunol. 2012; 129: 694-701.

116.- KOURI A, BOULET LP, KAPLAN A, GUPTA S. An evidence-based, point-of-care tool to guide completion of asthma action plans in practice. Eur Respir J. 2017; 49: 1602238. doi: 10.1183/13993003.02238-2016.

Correspondencia a:

Dra. Ana María Herrera Gana

Email: amherreragana@gmail.com 